\title{
Geometrisches Begriffsverständnis in der Grundschule am Beispiel der Begriffe Viereck, Rechteck und Quadrat
}

\author{
Julia Bruns $\mathbb{D} \cdot$ Elisabeth Unterhauser $\cdot$ Hedwig Gasteiger
}

Eingegangen: 28. September 2020 / Angenommen: 22. April 2021 / Online publiziert: 9. Juni 2021

(C) Der/die Autor(en) 2021

Zusammenfassung $\mathrm{Zu}$ den ersten geometrischen Begriffen, die Kinder bereits im Elementar- und Primarbereich lernen, zählen u. a. Viereck, Rechteck und Quadrat. Studien zeigen, dass Lernende bereits früh individuelle Vorstellungen, sog. individuelle Begriffskonzepte, zu diesen Begriffen aufbauen. Zwar wird die Entwicklung von Begriffsverständnis in verschiedenen mathematikdidaktischen Stufenmodellen dargestellt, diese sind jedoch generisch und beschreiben nicht explizit die Entwicklung der ersten individuellen Begriffskonzepte von Lernenden zu Viereck, Rechteck und Quadrat. Aus empirischer Sicht liegen verschiedene Studien vor, die einzelne Aspekte der individuellen Begriffskonzepte von Lernenden unterschiedlicher Altersgruppen zu diesen Begriffen ausleuchten. Um Begriffsbildungsprozesse aus empirischer Sicht detaillierter entlang der jeweils vorherrschenden individuellen Begriffskonzepte zu beschreiben, fehlen insbesondere Studien in der Grundschule, die alle vier Klassenstufen betrachten und dabei differenzierte Erkenntnisse zu verschiedenen theoretischen Indikatoren des Begriffsverständnisses liefern. Daher geht die vorliegende Studie der Frage nach, welches Verständnis der Begriffe Viereck, Rechteck und Quadrat Schülerinnen und Schüler der Jahrgangsstufen 1, 2, 3 und 4 zeigen. Dazu wurde eine Quasi-Längsschnittstudie mit $N=456$ Grundschulkindern (ca. 100 pro Jahrgangsstufe) durchgeführt. Die Ergebnisse geben detaillierte Einblicke in die individuellen Begriffskonzepte der Lernenden und zeigen, dass Lernende zunehmend Eigenschaften der Figuren berücksichtigen, jedoch individuelle Begriffskonzepte über lange Zeit auch prototypisch geprägt sind. Implikationen dieser Ergebnisse für Forschung und Praxis werden diskutiert.

Julia Bruns $(\bowtie)$

Institut für Mathematik (Mathematikdidaktik), Universität Paderborn,

Warburgerstraße 100, 33098 Paderborn, Deutschland

E-Mail: julia.bruns@uni-paderborn.de

Elisabeth Unterhauser · Hedwig Gasteiger

Institut für Mathematik (Mathematikdidaktik), Universität Osnabrück, Osnabrück, Deutschland 
Schlüsselwörter Begriffsverständnis · Geometrie · Grundschule · Viereck · Rechteck · Quadrat

\title{
Understanding of Geometrical Concepts in Elementary School Using the Example of Quadrangle, Rectangle and Square
}

\begin{abstract}
Among the first geometric concepts that children learn in early childhood education and primary school are quadrangle, rectangle and square. Studies show that learners have already built up individual definitions, so-called personal figural concepts, for these concepts at an early age. The development of this understanding of geometrical concepts is presented in theories that describe different levels. These models are, however, relatively generic and do not describe the personal figural concepts of learners concerning quadrangle, rectangle and square. From an empirical point of view, there are several studies that shed light on aspects of the personal figural concepts of learners of different age groups. In order to describe the development of geometrical understanding from an empirical point integrating the personal figural concepts, however, studies in primary school that consider all four class levels and provide insights into theoretical indicators of conceptual understanding are particularly lacking. In a first step, the present study therefore pursues the following question: Which understanding of the geometrical concepts 'quadrangle', 'rectangle' and 'square' show primary school students in the classes 1, 2, 3 and 4? A quasi-longitudinal study with $N=456$ students (approx. 100 in each class level) was conducted. The results give detailed insights into the personal figural concepts of the learners and show that learners increasingly consider the characteristics of these concepts, but that personal figural concepts are also prototypical over a long period of time. Implications for future research and practice are discussed.
\end{abstract}

Keywords Conceptual knowledge $\cdot$ Geometry $\cdot$ Elementary school $\cdot$ Quadrangle · Rectangle $\cdot$ Square

\section{Einleitung}

Kinder lernen bereits im Elementar- und Primarbereich verschiedene geometrische Begriffe kennen; zu den Ersten zählen u. a. Viereck, Rechteck und Quadrat. Studien mit Kindern aus dem Elementarbereich und zu Schulbeginn (Aktaş Arnas und Aslan 2007; Grassmann et al. 2002; Höglinger und Senftleben 1997; Unterhauser 2020) zeigen, dass Lernende bereits früh individuelle Begriffskonzepte, sog. personal figural concepts (Fujita und Jones 2007), zu diesen Begriffen aufbauen. Die (Weiter-)Entwicklung dieses Begriffsverständnisses wird innerhalb der Mathematikdidaktik in sog. Stufenmodellen durch qualitative Unterschiede bezüglich verschiedener Indikatoren beschrieben (van Hiele 1986; Vollrath 1984; Winter 1983). Da es sich jedoch um generische Stufenmodelle zur Begriffsbildung handelt, bleiben diese hinsichtlich individueller Begriffskonzepte der Lernenden zu Vierecksbegriffen eher abstrakt. Fujita und Jones (2007) weisen darauf hin, dass Studien zu individuellen Begriffskonzepten und deren Abweichungen von den formal-logischen Begriffskon- 
zepten (i. O. formal figural concepts), den mathematisch korrekten Definitionen, didaktisch von besonderem Interesse sind, da gerade die Abweichungen dazu beitragen können, Hypothesen über Begriffsbildungsprozesse zu bilden. Verschiedene empirische Studien untersuchen individuelle Begriffskonzepte zu Viereck, Rechteck und Quadrat von Lernenden unterschiedlicher Altersstufen (Aktaş Arnas und Aslan 2010; Fujita 2012; Fuys et al. 1988; Grassmann et al. 2002; Heinze 2002a, b; Höglinger und Senftleben 1997; Unterhauser 2020). Ergebnisse weisen darauf hin, dass Schülerinnen und Schüler auch in der Sekundarstufe noch Schwierigkeiten mit dem Verständnis dieser Begriffe haben (Burger und Shaughnessy 1986; Fujita 2012; Fuys et al. 1988; Heinze 2002a, b). Gleichzeitig zeigen sich nach umfassender Recherche Forschungslücken bezüglich des Begriffsverständnisses von Viereck, Rechteck und Quadrat in der Primarstufe, insbesondere hinsichtlich Studien, die auf allen Indikatoren des Begriffsverständnis basieren (s. Abschn. 2). Hier setzt die vorliegende Studie an, mit dem Ziel eine detaillierte Analyse der individuelle Begriffskonzepte vorzunehmen, um Hypothesen zu Begriffsbildungsprozessen zu generieren.

\section{Formal-logische Definition der Begriffe Viereck, Rechteck und Quadrat}

Für den Begriff Viereck gilt: Befinden sich vier Punkte in allgemeiner Lage, d.h. keine drei Punkte liegen auf derselben Geraden, dann ist ein Viereck ein geschlossener Streckenzug, der vier Punkte durch vier Strecken miteinander verbindet. Die Punkte werden als Ecken des Vierecks bezeichnet, die Strecken als Seiten, die anderen Verbindungsstrecken als Diagonalen (Wellstein und Kirsche 2009). Auf Basis der Lage der Diagonalen lassen sich Viereckstypen unterscheiden: Bei konvexen Vierecken liegen beide Diagonalen innerhalb, bei konkaven liegt eine der Diagonalen außerhalb, bei überschlagenen Vierecken liegen beide Diagonalen außerhalb des Vierecks (Kürpig und Niewiadomski 1992). Die Definition des Begriffs Vierecks enthält alle sog. definierenden Eigenschaften (geschlossen, vier Ecken, vier Seiten), die alle Repräsentanten des Begriffs erfüllen müssen (Begriffsinhalt). Zusätzlich weisen Repräsentanten des Begriffs Viereck auch sog. nicht-definierende Eigenschaften (bspw. Orientierung parallel zum Blattrand, Seitenlänge, Winkelmaß) auf, die aus mathematischer Sicht irrelevant und nicht allen Repräsentanten gemein sind.

Aus der formal-logischen Definition ergibt sich die Menge der Figuren, die als Vierecke beschrieben werden kann (Begriffsumfang). Diese Menge enthält Teilmengen spezifischer Vierecke (bspw. Rechtecke), die wiederum Teilmengen von spezifischen Vierecken enthalten können (z. B. Quadrate). Die Beziehung zwischen Gesamt- und Teilmengen ist dadurch hierarchisch und wird als Klasseninklusion betitelt. Demgegenüber steht eine Betrachtung der Teilmengen als paarweise disjunkt, also als Partitionen der Gesamtmenge. Dieses Verständnis der Beziehung zwischen Gesamtmenge und Teilmengen (z.B. Quadrate, Rechtecke, die keine Quadrate sind) wird partitionale Klassifikation genannt.

Es besteht Einigkeit darüber, dass Lernende ein Verständnis der Klasseninklusion (bildlich als ,Haus der Vierecke‘ beschrieben) aufbauen sollen (Heinze 2002a), 
wobei dies nach unterschiedlichen Kriterien erfolgen kann. Wird ein Haus der Vierecke über die Eigenschaften von Seiten und Winkel aufgebaut, ergeben sich für die Begriffe Rechteck und Quadrat folgende Definitionen: Ein Rechteck ist ein Parallelogramm mit vier rechten Winkeln und ein Quadrat ein Rechteck mit vier gleich langen Seiten (Wellstein und Kirsche 2009). Es entsteht ein Begriffsnetz, bei dem der Begriff Viereck mit den wenigsten definierenden Eigenschaften der Oberbegriff ist. Um Unterbegriffe, wie beispielsweise Rechteck und Quadrat, zu definieren, kommen zu den Eigenschaften des Oberbegriffs jeweils begriffsspezifische definierende Eigenschaften hinzu.

Neben der formal-logischen Definition geometrischer Begriffe ist aus didaktischer Perspektive von Interesse, wie Lernende diese Begriffe verstehen. Dazu liegen verschiedene Theorien des Begriffsverständnisses vor.

\section{Klassische und prototypische Theorie des Begriffsverständnisses}

Geometrische Begriffe, darunter auch ebene Figurenbegriffe wie Viereck, Rechteck und Quadrat, sind laut der klassischen Theorie (Hull 1920; Medin und Smith 1984) strukturell durch Begriffsinhalt, -umfang, -wort und -netz definiert. Der Begriffsinhalt beschreibt die definierenden Eigenschaften. Geometrische Begriffe werden dadurch eindeutig definiert (siehe Abschn. 2). Der Begriffsumfang umfasst alle Objekte, die die definierenden Eigenschaften eines Begriffs erfüllen, die sog. Repräsentanten des Begriffs (Hischer 2012; Medin und Smith 1984). Das Begriffswort bezeichnet einen Begriff als symbolische Repräsentation in sprachlicher Form. Ein geometrischer Begriff steht nicht alleine, sondern ist Teil eines hierarchischen Begriffsnetzes aus Ober-, Neben- und Unterbegriffen (Franke und Reinhold 2016). Die klassische Theorie geht davon aus, dass geometrische Begriffe von Lernenden mit Hilfe dieser Indikatoren (Begriffsinhalt, -umfang, -wort und -netz) gespeichert werden.

Die prototypische Theorie folgt dagegen der Annahme, dass manche Repräsentanten, sog. Prototypen, als ,beste Beispiele“ eines Begriffs gespeichert werden und folglich schneller als Repräsentanten des Begriffs erkannt werden als andere (Medin und Smith 1984; Rosch 1978). Prototypische Vorstellungen entwickeln sich auf Grundlage der Erfahrungen zu Begriffen. Dabei werden in der Regel diejenigen Repräsentanten zu Prototypen, die besonders häufig mit dem Begriff verknüpft werden. Ein Prototyp ist meist durch eine Kombination definierender, aber auch nicht-definierender Eigenschaften, gekennzeichnet, die man charakteristische Eigenschaften nennt. Ein Prototyp für das Viereck wäre bspw. das Quadrat. Das Quadrat weist alle definierenden Eigenschaften auf und hat darüber hinaus vier rechte Winkel und gleichlange Seiten als nicht-definierende Eigenschaften. Charakteristische Eigenschaften eines Vierecks sind somit vier Ecken bzw. Seiten, gleichlange Seiten sowie (annährend) rechte Winkel. Ausgehend von charakteristischen Eigenschaften kann bestimmt werden, wie typisch (also prototypisch) oder untypisch (also nicht prototypisch) ein Repräsentant ist (Medin und Smith 1984; Rosch 1978; Schmid 2000).

In Bezug auf die Entwicklung des Begriffsverständnisses von Lernenden erweisen sich klassische und prototypische Theorien als bedeutsam: Während die klassische 
Theorie Indikatoren des Begriffsverständnisses aufzeigt, verweist die prototypische Theorie auf die Bedeutung der individuellen Sichtweisen von Lernenden, die dazu führen können, dass Lernende charakteristische Eigenschaften - und damit ggf. fälschlicherweise nicht-definierende - als definierende Eigenschaften des Begriffs verstehen (bspw. gleich lange Seiten beim Viereck). Im Folgenden werden Modelle zur Entwicklung des Begriffsverständnisses betrachtet, die u.a. diese Aspekte der klassischen und prototypischen Theorie berücksichtigen.

\section{Modelle zur Entwicklung des Begriffsverständnisses}

In Stufenmodellen zur Entwicklung des Begriffsverständnisses, bspw. von van Hiele (1986), Vollrath (1984) und Winter (1983), werden - basierend auf theoretischen Überlegungen zur Begriffsbildung und Erkenntnissen Piagets et al. (1974) verschiedene Charakteristika geometrischen Begriffsverständnisses ausdifferenziert. Insgesamt lassen sich in den verschiedenen, idealtypischen Modellen fünf inhaltlich ähnliche Stufen identifizieren (van Hiele 1986; Vollrath 1984; Winter 1983); maximal die ersten drei Stufen werden als relevant für die Primarstufe benannt (Franke und Reinhold 2016).

Idealtypisch wird auf der ersten Stufe ein Begriff ganzheitlich verstanden (bspw. Vergleiche zu Alltagsobjekten, Prototypen als mentale Bilder). Auf der nächsten Stufe operieren Lernende mit Eigenschaften des Begriffs, wobei die Eigenschaften noch isoliert nebeneinanderstehen. Auf dieser Stufe gewinnt das Verständnis des Begriffsinhalts an Bedeutung. Erst auf der Stufe des relationalen Begriffsverständnisses werden Beziehungen sowohl zwischen Eigenschaften als auch zwischen verschiedenen Begriffen hergestellt und es wird ein Begriffsnetz aufgebaut, d.h., ein Verständnis für die Klasseninklusion entwickelt sich (zusammenfassend s. a. Unterhauser 2020, S. $141 \mathrm{ff}$.).

Entwicklungspsychologische (Siegler 2006) und mathematikdidaktische (Battista 2007) Erkenntnisse zeigen, dass sich verschiedene Arten, geometrisch zu denken (bspw. ganzheitlich vs. eigenschaftsbezogen), zeitlich überlappen. Dabei wird angenommen, dass Kinder gleichzeitig über unterschiedliche Arten des Denkens bzw. Strategien verfügen, die miteinander konkurrieren und interagieren, und sich kontinuierlich verändern (Chen und Siegler 2000). Folglich wird davon ausgegangen, dass das ganzheitliche Denken nicht vom eigenschaftsbezogenen Denken abgelöst wird, sondern die unterschiedlichen Charakteristika geometrischen Denkens - im Sinne überlappender Wellen - parallel verlaufen können, aber auf unterschiedlichen Niveaus (bezeichnet als Wellenmodell). Welche Art des geometrischen Denkens jeweils Vorrang hat, kann aufgaben- bzw. kontextabhängig sein (Battista 2007; Clements et al. 1999; Fuys et al. 1988). Dabei stehen die beiden Modellvorstellungen (Stufen- und Wellenmodell) nicht im Widerspruch zueinander, sondern ergänzen sich: Während Stufenmodelle eher begriffsspezifisch hierarchisch unterschiedliche Charakteristika geometrischen Denkens skizzieren, bedienen sich Wellenmodelle dieser Denkweisen, um die Entwicklung des Begriffsverständnisses individuell zu beschreiben. 
Zur Analyse des individuellen Begriffsverständnisses von Lernenden schlagen Fujita und Jones (2007) eine Unterscheidung zwischen formal-logischen Begriffskonzepten, den Konzepten, die der mathematisch korrekten Definition eines Figurenbegriffs folgen, und individuellen Begriffskonzepten, die die individuellen Vorstellungen der Lernenden beschreiben, vor. Individuelle Begriffskonzepte werden von den Lernenden auf Grundlage ihrer Erfahrungen (bspw. im Geometrieunterricht) aufgebaut. Demnach kann das individuelle Begriffskonzept zu verschiedenen Vierecken neben den definierenden Eigenschaften weitere nicht-definierende Eigenschaften oder nicht alle definierenden Eigenschaften umfassen (s. a. Abschn. 3). Individuelle Begriffskonzepte zum Begriff Viereck könnten bspw. ausschließlich Figuren umfassen, die quadratisch sind, zum Begriff Rechteck ausschließlich Rechtecke, die ein Seitenverhältnis von 2:1 aufweisen und zum Begriff Quadrat alle Figuren, die gleich lange Seiten haben. Individuelle Begriffskonzepte beschreiben folglich Kriterien, die Lernende für den jeweiligen Begriff als bestimmend annehmen, beeinflussen also das Verständnis von Begriffsumfang, -inhalt und -netz gleichermaßen.

Da die Modellvorstellungen zur Entwicklung des Begriffsverständnisses aufgrund des generischen Anspruchs vergleichsweise abstrakt bleiben, sind die expliziten individuellen Begriffskonzepte zu Viereck, Rechteck und Quadrat in verschiedenen Altersbereichen Gegenstand verschiedener Studien.

\section{Forschungsstand zum geometrischen Begriffsverständnis}

Internationale Studien zum Verständnis ebener Figurenbegriffe untersuchen mehrheitlich Kinder im Elementarbereich (Aktaş Arnas und Aslan 2007; Clements et al. 1999; Halat und Yeşil-Dağl1 2016; Koleza und Giannisi 2013; Levenson et al. 2011; Maier 2019; Unterhauser und Gasteiger 2018; Unterhauser 2020), spezifisch zu Schuleintritt (Grassmann 1996; Grassmann et al. 2002; Höglinger und Senftleben 1997; Kyriakides 1999; Reemer und Eichler 2005) oder im Elementar- und Primarbereich kombiniert (Aktaş Arnas und Aslan 2010; Gagatsis et al. 2006; Gunčaga et al. 2017; Žilková und Kopáčová 2018). Für den Primarbereich konnten lediglich zwei qualitative Untersuchungen (Burger und Shaughnessy 1986; Lehrer et al. 1998) und eine quantitative (Ma et al. 2015) gefunden werden, die alle vier Klassenstufen berücksichtigen. Darüber hinaus existieren Studien, die das Verständnis ebener Figurenbegriffe von Lernenden in der Sekundarstufe (Burger und Shaughnessy 1986; Fuys et al. 1988; Fujita 2012; Heinze 2002a, b) erfassen.

Ein großer Teil der empirischen Studien untersucht die Begriffe Rechteck und Quadrat (bspw. Aktaş Arnas und Aslan 2010; Clements et al. 1999; Gagatsis et al. 2006). Der Begriff Viereck wird als Oberbegriff primär in Studien aus dem deutschsprachigen Raum untersucht (Grassmann et al. 2002; Heinze 2002a; Höglinger und Senftleben 1997; Reemer und Eichler 2005; Unterhauser 2020, Ausnahme: Ma et al. 2015). Im Folgenden wird zunächst die Figurenauswahl, die in Studien zur Untersuchung des Begriffsverständnisses zu Viereck, Rechteck und Quadrat häufig eingesetzt wurde, näher erläutert, ehe vorliegende empirische Erkenntnisse strukturiert nach den Begriffen sowie Erkenntnisse zum Verständnis der Klasseninklusion dargestellt werden. 


\subsection{Typische und untypische Repräsentanten bzw. Nicht-Repräsentanten}

Zur Operationalisierung des Begriffsverständnisses nutzen Studien mit Lernenden im Elementar-, Primar- und Sekundarbereich häufig Aufgaben zum Sortieren, Identifizieren (Begriffsumfang) sowie zum Definieren bzw. Erklären und Begründen (Begriffsinhalt). Werden Lernende zum Sortieren oder Identifizieren aufgefordert, werden ihnen verschiedene Figuren präsentiert. Aufbauend auf die wiederholt verwendete Figurenauswahl von Razel und Eylon (1991) und empirischen Erkenntnissen zu Schwierigkeiten von Lernenden konstruierten Aslan und Aktaş Arnas (2007) eine in den definierenden und nicht-definierenden Eigenschaften systematisch variierte Figurenauswahl, u. a. für die Begriffe Rechteck und Quadrat (vgl. Abb. 1a, b).

Für die Repräsentanten des jeweiligen Figurenbegriffs unterscheiden Aslan und Aktaş Arnas (2007) - angelehnt an die prototypische Theorie (vgl. Abschn. 2) - typische Repräsentanten (bspw. R3 für den Begriff Rechteck) und untypische Repräsentanten (bspw. R5 für den Begriff Rechteck), welche zusätzliche, nicht-definierende Eigenschaften aufweisen und/oder in der Lage bzw. Größe stark von den typischen Repräsentanten abweichen (bspw. Abb. 1b, S1 vs. S3). Nicht-Repräsentanten des jeweiligen Figurenbegriffs unterscheiden Aslan und Aktaş Arnas (2010) ebenfalls in typische (bspw. RD3 für den Begriff Rechteck) und untypische Nicht-Repräsentanten (bspw. RD1 für den Begriff Rechteck). Untypische Nicht-Repräsentanten ähneln dabei typischen Repräsentanten aus ganzheitlicher Perspektive, ihnen fehlen allerdings definierende Eigenschaften, wohingegen typische Nicht-Repräsentanten

Abb. 1 Identifikationsfiguren für die Begriffe Rechteck (a), Quadrat (b) (Aslan und Aktaş Arnas 2007, S. 101-103) und Viereck (c) (Unterhauser 2020, S. 270) a

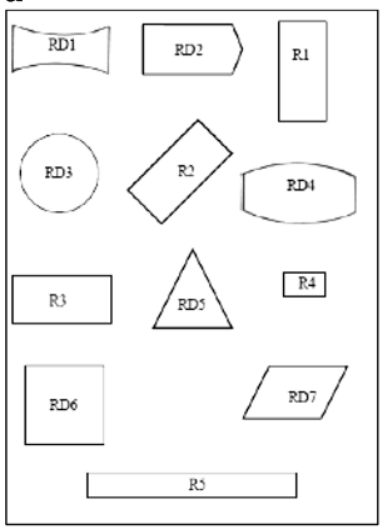

b

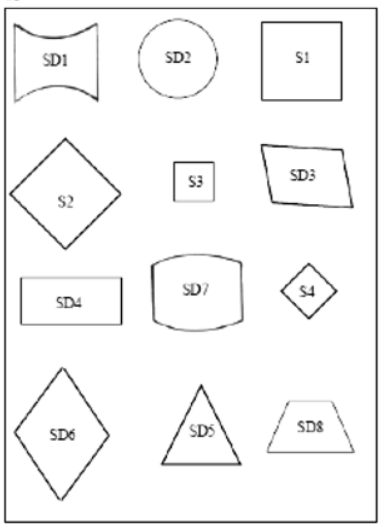

C

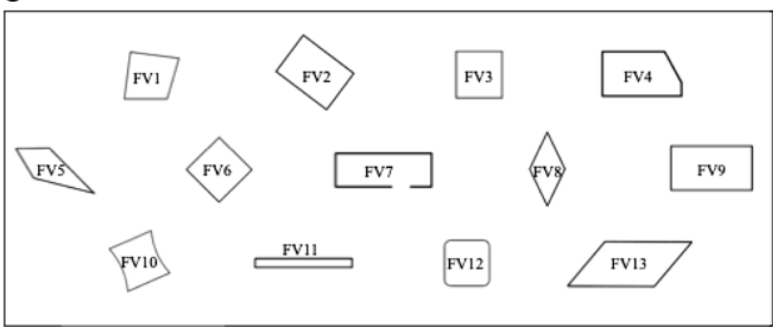


aus ganzheitlicher Sicht keine Ähnlichkeit mit typischen Repräsentanten haben und nicht alle definierenden Eigenschaften aufweisen. Die dargestellte bzw. eine ähnliche Auswahl von Figuren wird in Studien zum Verständnis der Begriffe Rechteck und Quadrat sowohl mit Lernenden im Elementarbereich (Aktaş Arnas und Aslan 2007) als auch im Primarbereich (Aktaş Arnas und Aslan 2010) genutzt.

Eine systematisch variierte Figurenauswahl für den Begriff Viereck konstruierte Unterhauser (2020) in ähnlicher Weise für ihre Studie mit Kindern zwischen vier und sechs Jahren. So zeigt Abb. 1c bspw. ein Quadrat (FV3) als typischen Repräsentanten des Begriffs Viereck, eine Raute (FV8) als untypische Repräsentanten und ein Fünfeck (FV4) als Nicht-Repräsentanten. Die Figurenauswahl wurde jedoch bislang nicht für Studien mit Lernenden im Primarbereich erprobt.

\subsection{Verständnis des Begriffs Viereck}

In nahezu allen Studien zum Verständnis des Begriffs Viereck wird eine Stichprobe von Kindern im Elementarbereich bzw. im Übergang zur Grundschule untersucht, nur die Studie von Ma et al. (2015) untersucht ausschließlich Lernende im Primarbereich.

Die Ergebnisse der Studien aus dem Elementarbereich lassen erkennen, dass Erklärungen der Kinder für den Begriff Viereck sich oft auf die Anzahl der Ecken und/ oder Seiten stützen. Gleichzeitig enthalten die Erklärungen informelle bzw. alltagssprachliche Elemente (bspw. Vergleiche mit Alltagsobjekten) oder nicht-definierende Eigenschaften (Reemer und Eichler 2005; Unterhauser 2020). Teilweise stehen die Erklärungen der Begriffe und die getroffenen Identifikationen nicht im Einklang: Kinder geben bspw. an, dass ein Viereck vier Ecken haben muss, akzeptieren aber dennoch lediglich Quadrate als Repräsentanten des Begriffs Viereck (ebd.).

Als Repräsentanten des Begriffs Viereck akzeptieren Kinder in erster Linie Quadrate, obwohl ein geringer Prozentsatz auch Rechtecke identifiziert (Grassmann et al. 2002; Höglinger und Senftleben 1997; Reemer und Eichler 2005; Unterhauser 2020). Mitunter werden ein prototypisches Quadrat und das Begriffswort, Quadrat' als Synonym für den Begriff Viereck verstanden (Grassmann 1996; Grassmann et al. 2002; Reemer und Eichler 2005; Unterhauser 2020; Unterhauser und Gasteiger 2018). Insgesamt werden mehr typische als untypische Repräsentanten korrekt identifiziert und Nicht-Repräsentanten (bspw. FV12) tendenziell eher fälschlicherweise als Repräsentanten des Begriffs Viereck aufgefasst (Unterhauser 2020).

Hinsichtlich des Verständnisses der Eigenschaften des Begriffs Viereck zeigt sich, dass - obwohl nicht-definierend - bspw. Winkel, Seitenlängen und die Orientierung parallel zum Blattrand der Figur beeinflussen, ob eine Figur als Viereck identifiziert wird. Lernende in der Primarstufe zeigen vor allem Probleme mit der Identifikation von stumpfwinkligen sowie konkaven Vierecken (Ma et al. 2015). Bei den definierenden Eigenschaften hat die ,Offenheit einer Figur ${ }^{61}$ zur Folge, dass eine Figur

\footnotetext{
1 Aus mathematischer Perspektive handelt es sich bei dem Beispiel FV7 nicht um eine Figur, sondern um einen offenen Polygonzug. Im Folgenden wird dennoch für Objekte dieser Art der Begriff offene Figur bzw. offene Seite verwendet, um zu verdeutlichen, dass das jeweilige Objekt für die Untersuchung ausgewählt wurde, um zu untersuchen, inwieweit die Geschlossenheit des Streckenzugs als Eigenschaft einer (Vierecks-)Figur erkannt wird.
} 
(bspw. FV7) in der Regel korrekterweise nicht als Repräsentant identifiziert wird (ebd.); quadratähnliche Figuren mit abgerundeten ,Ecken`2 (bspw. FV12) werden allerdings eher fälschlicherweise als Repräsentant identifiziert als quadratähnliche Figuren mit gebogenen ,Seiten“ (bspw. FV10) (Unterhauser 2020). Insgesamt zeigt sich jedoch auch, dass Grundschülerinnen und Grundschüler in höheren Klassenstufen mehr Figuren zum Begriff Viereck korrekt identifizieren als Kindern in niedrigeren Klassenstufen (Ma et al. 2015).

\subsection{Verständnis der Begriffe Rechteck und Quadrat}

Für die Begriffe Rechteck und Quadrat formulieren Kinder Erklärungen, die neben der Anzahl der Ecken bzw. Seiten auch informelle bzw. alltagssprachliche Informationen beinhalten (Hannibal 1999; Levenson et al. 2011; Maier 2019; Reemer und Eichler 2005). Selbst Schülerinnen und Schüler in der Sekundarstufe I oder darüber hinaus haben Schwierigkeiten, zwischen hinreichenden und notwendigen Bedingungen zu unterscheiden und können gleichwertige Definitionen, zum Beispiel für den Begriff Quadrat, nur schwer als solche erkennen (Burger und Shaughnessy 1986; Fujita 2012; Heinze 2002a).

Als Repräsentanten für die Begriffe Rechteck und Quadrat zeichnen Kinder in erster Linie Prototypen: für den Begriff Rechteck ein Rechteck in horizontaler Orientierung parallel zum Blattrand mit einem Seitenverhältnis von etwa 2:1 und für den Begriff Quadrat ein Quadrat parallel zum Blattrand (Halat und Yeşil-Dağlı 2016; Kyriakides 1999). Ergebnisse bisheriger Studien mit Kindern im Elementarbereich zeigen bei Aufgaben zum Identifizieren ohne untypische Nicht-Repräsentanten für die Begriffe Rechteck und Quadrat mit ca. 75-85\% hohe Identifikationsraten, wobei für den Begriff Quadrat in der Regel mehr korrekte Identifikationen getroffen werden (Clements et al. 1999; Kyriakides 1999; Reemer und Eichler 2005). Insgesamt treffen Schülerinnen und Schüler der Klasse 4 im Vergleich zu Kindern im Elementarbereich für die Begriffe Rechteck (bspw. $91 \%$ Klasse 4 vs. 58\% 4-Jährige) und Quadrat (bspw. $91 \%$ Klasse 4 vs. $81 \%$ 4-Jährige) mehr korrekte Identifikationen (bspw. Aktaş Arnas und Aslan 2010). Allerdings sind die Unterschiede zwischen den Altersgruppen nicht durchweg signifikant, was mitunter daran liegt, dass jüngere Kinder auch untypische Repräsentanten (bspw. Abb. 1, S4) häufiger als Repräsentanten akzeptieren als ältere Kinder (Aktaş Arnas und Aslan 2010; Žilková und Kopáčová 2018). Sowohl für den Begriff Rechteck als auch den Begriff Quadrat werden bei den vorliegenden Studien mit Kindern im Elementarbereich untypische Nicht-Repräsentanten (bspw. Abb. 1, RD1) weniger häufig korrekt identifiziert als typische Nicht-Repräsentanten (bspw. Abb. 1, RD5) (Aslan und Aktaş Arnas 2007; Maier 2019).

\footnotetext{
2 Aus mathematischer Perspektive handelt es sich weder bei Beispiel FV12 noch FV10 um Polygone, da diese Objekte aus mathematischer Perspektive keine Ecken bzw. Seiten besitzen. Im Folgenden werden für Objekte dieser Art dennoch die Begriffe ,Ecke‘ bzw. ,Seite` genutzt, um zu verdeutlichen, dass das jeweilige Objekt für die Untersuchung ausgewählt wurde, um zu untersuchen, inwieweit die Begriffe Ecke bzw. Seite bereits mathematisch korrekt verstanden werden.
} 
Hinsichtlich der Rolle von nicht-definierenden Eigenschaften bei der Identifikation von Repräsentanten zeigt sich für den Begriff Rechteck, dass die Orientierung parallel zum Blattrand einer Figur wenig Einfluss auf die korrekte Identifikationsentscheidung hat (ebd.). Für den Begriff Quadrat scheint die Orientierung parallel zum Blattrand dagegen eine Rolle zu spielen: Steht ein Quadrat auf der Ecke, fällt die Identifikationsrate von $90 \%$ (,Normalorientierung parallel zum Blattrand ') auf 60 bis $70 \%$, nicht nur in Studien mit Kindern aus dem Elementarbereich (Elementarbereich: Aslan und Aktaş Arnas 2007; Clements et al. 1999; Halat und YeşilDağlı 2016; Maier 2019; Elementar- und Primarbereich: Gunčaga und Žilková 2019; Primarbereich: Burger und Shaughnessy 1986; Yin 2003). Die Größe eines Repräsentanten hat hingegen kaum Einfluss auf die Identifikationsrate (Aktaş Arnas und Aslan 2010; Clements et al. 1999), außer die Figurenauswahl ist sehr eingeschränkt (Halat und Yeşil-Dağlı 2016; Žilková und Kopáčová 2018).

In Bezug auf die definierenden Eigenschaften zeichnet sich für den Begriff Rechteck ab, dass die Parallelität der Seiten von Kindern verschiedener Altersstufen höher gewichtet wird als die Rechtwinkligkeit (Aktaş Arnas und Aslan 2010; Burger und Shaughnessy 1986; Clements et al. 1999; Maier 2019; Žilková und Kopáčová 2018). Für den Begriff Quadrat scheinen die vier gleich langen Seiten entscheidend. In vielen Studien wird die Raute (vorwiegend von Kindern im Elementarbereich) als Repräsentant des Begriffs Quadrat identifiziert (Aslan und Aktaş Arnas 2007; Clements et al. 1999; Gunčaga et al. 2017; Koleza und Giannisi 2013). Ebenso wie für den Begriff Viereck, kann aus den Identifikationen für die Begriffe Rechteck und Quadrat geschlussfolgert werden, dass gebogene ,Seiten' bzw. abgerundete ,Ecken “ nicht immer dazu führen, dass die Figur als Repräsentant verworfen wird, sofern die Form einem typischen Repräsentanten ähnelt. Allerdings akzeptieren Grundschülerinnen und Grundschüler im Vergleich zu Kindern im Elementarbereich derartige Figuren vermehrt korrekterweise nicht als Repräsentant (Aslan und Aktaş Arnas 2007; Clements et al. 1999; Gunčaga et al. 2017; Reemer und Eichler 2005; Žilková und Kopáčová 2018).

Werden Kinder dazu aufgefordert, ihre Identifikationsentscheidungen für die Begriffe Rechteck und Quadrat zu begründen, dann führen sie hauptsächlich (70-80\%) ganzheitliche Argumente (bspw. ,Es sieht (nicht) aus wie ein Rechteck.') an, aber auch eigenschaftsbezogene, die bei Grundschülerinnen und Grundschülern tendenziell häufiger vorkommen als bei Kindern im Elementarbereich (Aktaş Arnas und Aslan 2010; Clements et al. 1999). Der Großteil der eigenschaftsbezogenen Begründungen bezieht sich auf die Anzahl der Ecken bzw. der Seiten (Aslan und Aktaş Arnas 2007; Clements et al. 1999; Grassmann 1996; Lehrer et al. 1998). Auch die Länge der Seiten - eine definierende Eigenschaft für den Begriff Quadrat, nicht aber für den Begriff Rechteck - wird als eigenschaftsbezogene Begründung für beide Begriffe angeführt (Aktaş Arnas und Aslan 2010; Clements et al. 1999; Reemer und Eichler 2005). 


\subsection{Verständnis der Klasseninklusion der Begriffe Viereck, Rechteck und Quadrat}

Studien aus dem deutschsprachigen Raum zeigen, dass junge Kinder den Begriff Viereck partitional klassifizieren (Grassmann et al. 2002; Höglinger und Senftleben 1997; Unterhauser 2020). Vor allem die Begriffe Quadrat und Rechteck werden in verschiedenen Altersstufen bis in die Sekundarstufe als voneinander getrennte Begriffe verstanden (Burger und Shaughnessy 1986; Clements et al. 1999; Fuys et al. 1988; Heinze 2002a, b; Höglinger und Senftleben 1997; Koleza und Giannisi 2013; Maier 2019; Reemer und Eichler 2005; Unterhauser 2020; Vinner und Hershkowitz 1980; Yin 2003). Für junge Kinder gilt meist: Selbst, wenn sie in ihrer Erklärung richtig angeben, dass ein Viereck vier Ecken bzw. Seiten haben muss, klassifizieren sie dennoch partitional und identifizieren bspw. ein Trapez nicht als Viereck (Höglinger und Senftleben 1997; Reemer und Eichler 2005). Zudem sehen Kinder im Elementarbereich, die mit den Eigenschaften des Begriffs Rechteck vertraut sind, Rechteck und Viereck als unabhängige Begriffe an (Grassmann et al. 2002; Höglinger und Senftleben 1997; Reemer und Eichler 2005; Unterhauser 2020). Wenngleich 4-jährige Kinder im Gegensatz zu anderen Altersgruppen für den Begriff Rechteck mitunter Quadrate als Rechtecke akzeptieren, werden beide Begriffe primär als disjunkt verstanden (Clements et al. 1999; Hannibal 1999; Unterhauser 2020). Unterrichtsversuche, wie bspw. von Bartolini Bussi und Baccaglini-Frank (2015), zeigen jedoch, dass es im Unterricht möglich ist, Kinder zu unterstützen, ein inklusives Verständnis der Begriffe Rechteck und Quadrat zu entwickeln.

\subsection{Zusammenfassung der Erkenntnisse zu den Begriffen Viereck, Rechteck und Quadrat}

Insgesamt zeigen die vorliegenden Erkenntnisse zu den Begriffen Viereck, Rechteck und Quadrat, dass begriffsspezifisch unterschiedliche Eigenschaften einen potenziellen Einfluss auf die Identifikationsentscheidungen von Kindern im Elementar- und Primarbereich haben. Verschiedene Studien haben systematische Figurenauswahlen konstruiert (bspw. Satlow und Newcombe 1998; Tsamir et al. 2008; Unterhauser 2020). Stellt man die figurenbezogenen Erkenntnisse gegenüber, dann lassen sich in Übereinstimmung mit der prototypischen Theorie (s. Abschn. 3) definierende, nicht-definierende und charakteristische Eigenschaften (s. Tab. 1) sowie aufbauend typische und untypische Repräsentanten bzw. Nicht-Repräsentanten für die Begriffe Viereck, Rechteck und Quadrat unterscheiden (s. Tab. 2).

Zusammenfassend werden für die Begriffe Viereck, Rechteck und Quadrat mehr korrekte Identifikationsentscheidungen für Nicht-Repräsentanten getroffen als für Repräsentanten, wobei es älteren Kindern im Vergleich zu jüngeren Kindern tendenziell besser gelingt, Nicht-Repräsentanten korrekt als solche zu identifizieren. Die ,typischen Repräsentanten“ in Tab. 2 sind die Prototypen der drei Begriffe. Ähnelt die Figur in ihrer Ganzheit einem Prototyp, hat aber bspw. runde ,Ecken“ (bspw. V17), gehört also zur Gruppe der untypischen Nicht-Repräsentanten, wird sie von Kindern in der Regel fälschlicherweise als Repräsentant identifiziert. Innerhalb der beiden Figurengruppen Repräsentanten und Nicht-Repräsentanten werden jeweils die typi- 
Tab. 1 Definierende, nicht-definierende und charakteristische Eigenschaften der Begriffe Viereck, Rechteck und Quadrat

\begin{tabular}{|c|c|c|c|}
\hline & Viereck & Rechteck & Quadrat \\
\hline $\begin{array}{l}\text { Definierende } \\
\text { Eigen- } \\
\text { schaften }\end{array}$ & $\begin{array}{l}\text { Geschlossene Figur } \\
4 \text { Ecken } \\
4 \text { Seiten }\end{array}$ & $\begin{array}{l}\text { Geschlossene Figur } \\
4 \text { Ecken } \\
4 \text { Seiten } \\
2 \text { Paar parallele Seiten } \\
4 \text { rechte Winkel }\end{array}$ & $\begin{array}{l}\text { Geschlossene Figur } \\
4 \text { Ecken } \\
4 \text { Seiten } \\
2 \text { Paar parallele Seiten } \\
4 \text { rechte Winkel } \\
4 \text { gleich lange Seiten }\end{array}$ \\
\hline $\begin{array}{l}\text { Nicht-de- } \\
\text { finierende } \\
\text { Eigen- } \\
\text { schaften }\end{array}$ & $\begin{array}{l}\text { Orientierung } \\
\text { Seitenlänge } \\
\text { Winkelmaß } \\
\ldots\end{array}$ & $\begin{array}{l}\text { Orientierung } \\
\text { Seitenlänge } \\
\ldots\end{array}$ & $\begin{array}{l}\text { Orientierung } \\
\ldots\end{array}$ \\
\hline $\begin{array}{l}\text { Charakteris- } \\
\text { tische } \\
\text { Eigen- } \\
\text { schaften }\end{array}$ & $\begin{array}{l}4 \text { Ecken } \\
4 \text { Seiten } \\
\text { Winkel ca. } 90^{\circ} \\
\text { Seitenlängenverhältnis } \\
2: 1 \text { oder } 1: 1 \\
\text { Orientierung parallel zu } \\
\text { Blattrand }\end{array}$ & $\begin{array}{l}4 \text { Ecken } \\
4 \text { Seiten } \\
4 \text { Winkel ca. } 90^{\circ} \\
\text { Gegenüberliegende Seiten } \\
\text { gleich lang } \\
\text { Seitenlängenverhältnis } 2: 1 \\
\text { Orientierung parallel zu Blat- } \\
\text { trand }\end{array}$ & $\begin{array}{l}4 \text { Ecken } \\
4 \text { Seiten } \\
\text { Winkel ca. } 90^{\circ} \\
4 \text { gleich lange Seiten } \\
\text { Orientierung parallel zu } \\
\text { Blattrand }\end{array}$ \\
\hline
\end{tabular}

Tab. 2 Übersicht typischer und untypischer Repräsentanten und Nicht-Repräsentanten verschiedener Studien

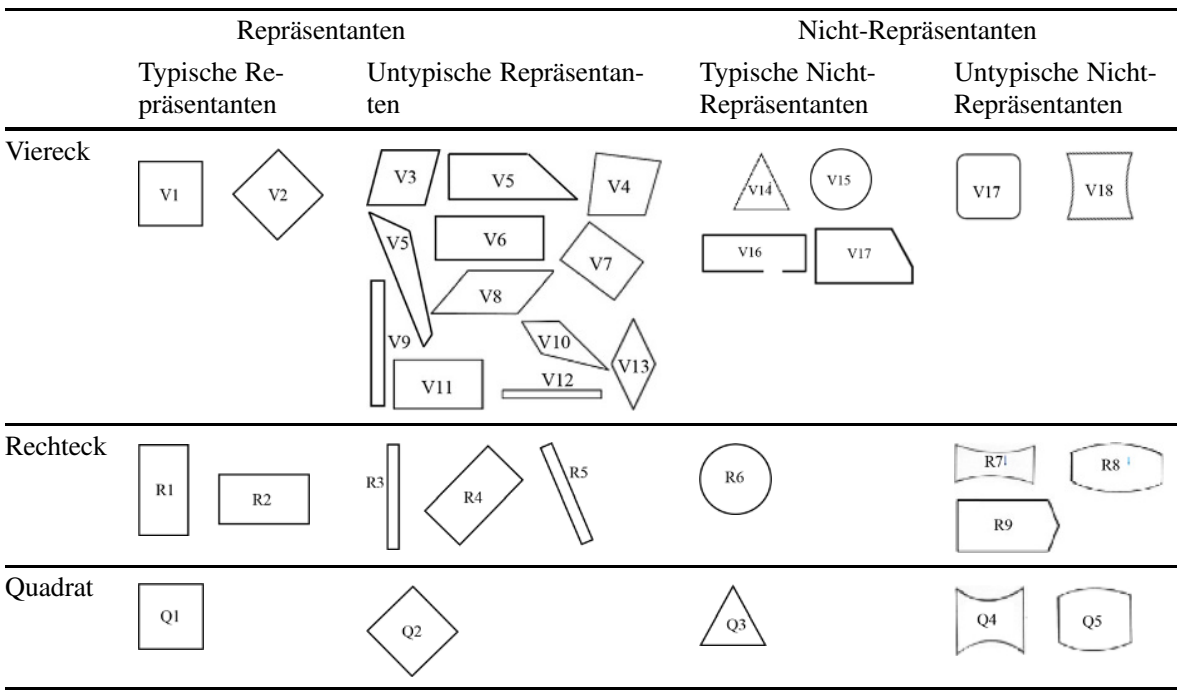

schen Figuren häufiger korrekt identifiziert als die untypischen. Wenngleich ältere Kinder tendenziell mehr korrekte Identifikationsentscheidungen treffen, so identifizieren jüngere Kinder mitunter untypische Repräsentanten eher korrekterweise als Repräsentanten. Identifikationsentscheidungen des Begriffs Viereck werden neben der Orientierung parallel zum Blattrand auch durch Winkelmaß und Seitenlänge beeinflusst - allesamt nicht-definierende Eigenschaft des Begriffs Viereck - wodurch bspw. untypische Repräsentanten (bspw. V10) nicht als Viereck identifiziert werden. 
Sowohl für den Begriff Rechteck als auch den Begriff Quadrat akzeptieren Kinder Figuren wie V3 oder V8 fälschlicherweise als Repräsentanten und scheinen für den Begriff Rechteck eher auf die Parallelität der zwei Seitenpaare zu achten bzw. für den Begriff Quadrat auf die gleiche Länge aller vier Seiten. Während ein Quadrat, dessen Seiten nicht parallel zum Blattrand ausgerichtet sind (bspw. Q2), seltener als Repräsentant des Begriffs Quadrat identifiziert wird, spielt die Orientierung parallel zum Blattrand bei Identifikationsentscheidungen für den Begriff Rechteck eine untergeordnete Rolle.

Werden Kindergarten- oder Grundschulkinder dazu aufgefordert, den Begriff Viereck, Rechteck und/oder Quadrat zu erklären, geben sie in erster Linie die Anzahl der Ecken und/oder Seiten als Eigenschaft an oder beziehen Vergleiche zu Alltagsobjekten in ihre Erklärungen ein. Grundschulkinder verwenden insgesamt weniger ganzheitliche Erklärungen und bei den Begriffen Rechteck und Quadrat wird auch die Seitenlänge als Eigenschaft erwähnt. Die charakteristischen Eigenschaften der Begriffe Viereck, Rechteck und Quadrat (s. Tab. 1) dominieren das Begriffsverständnis im Elementar- und Primarbereich. Außerdem werden die Begriffe Viereck, Rechteck und Quadrat zumeist als voneinander unabhängig verstanden, d.h., sowohl im Elementar- als auch im Primarbereich fehlt ein Verständnis für die Klasseninklusion.

\section{Herleitung und Begründung der Fragestellung}

Die dargestellten Theorien zur Entwicklung des (geometrischen) Begriffsverständnisses gehen davon aus, dass Lernende individuelle Begriffskonzepte zu ebenen Figurenbegriffen entwickeln, die (zunächst) nicht mit den formalen Definitionen dieser Begriffe übereinstimmen. Erste geometrische Begriffe, die in der Primarstufe eingeführt werden, sind u. a. die Begriffe Viereck, Rechteck und Quadrat (Franke und Reinhold 2016). Während zum Verständnis der Begriffe Rechteck und Quadrat verschiedene Studien vorliegen (bspw. Aktaş Arnas und Aslan 2010; Clements et al. 1999; Maier 2019), sind Studien - auch aufgrund der Unterschiede zwischen den Begriffen in verschiedenen Sprachen - zum Verständnis des Begriffs Viereck eher selten (Höglinger und Senftleben 1997; Reemer und Eichler 2005; Unterhauser 2020). Bisherige empirische Ergebnisse zum Verständnis der Begriffe Viereck, Rechteck und Quadrat stützen theoretische Überlegungen zum gestuften Erwerb des Begriffsverständnisses grundsätzlich und weisen auf spezifische Schwierigkeiten insbesondere bezüglich eines eigenschaftsbezogenen Verständnisses dieser Begriffe hin (s. a. Abschn. 5). Dabei scheinen Erkenntnisse zu einzelnen Indikatoren des Begriffsverständnisses (Begriffsinhalt, -umfang und -netz) in Bezug auf die Begriffe Viereck, Rechteck und Quadrat von Grundschülerinnen und -schülern nach ausführlicher Recherche bislang zu fehlen. Diese hätten jedoch das Potenzial, ein gezieltes Identifizieren individueller Begriffskonzepte zu erleichtern und, über generische Stufenmodelle hinaus, Hypothesen zum Erwerb des Begriffsverständnisses entlang dieser drei Indikatoren zu entwickeln. Diese Erkenntnisse könnten auch erklärende Hinweise zu Schwierigkeiten im Begriffsverständnis älterer Lernender geben und 
Grundlagen für Empfehlungen zur Gestaltung von Begriffsbildungsprozessen in der Grundschule bieten.

Folglich wird in dieser Studie der Vergleich des Begriffsverständnisses zu Viereck, Rechteck und Quadrat zwischen den vier Klassenstufen der Grundschule entlang der Indikatoren des Begriffsverständnisses fokussiert. Daraus ergibt sich die leitende Fragestellung:

Welches Verständnis der Begriffe Viereck, Rechteck und Quadrat zeigen Grundschülerinnen und Grundschüler der Jahrgangsstufen 1, 2, 3 und 4? Ziel der Studie ist einerseits die Deskription der individuellen Begriffskonzepte zu Viereck, Rechteck und Quadrat in den einzelnen Klassenstufen der Grundschule sowie eine explorative Suche nach Unterschieden zwischen den Klassenstufen, auf deren Basis Hypothesen über Begriffsbildungsprozesse formuliert werden können. Die Untersuchung des Begriffsverständnisses zu den Begriffen Viereck, Rechteck und Quadrat erfolgt in Anlehnung an die klassische Theorie (siehe Abschn. 3), entlang der Indikatoren Begriffsinhalt, -umfang und -netz.

\section{Forschungsdesign}

\subsection{Stichprobe}

Zur Untersuchung der Forschungsfrage wurde eine Quasi-Längsschnittstudie durchgeführt. Die Gelegenheitsstichprobe umfasste $N=456$ Schülerinnen und Schüler ( $n=217$ männlich, $n=238$ weiblich, $n=1$ ohne Angabe) drei verschiedener Grundschulen (zwei Niedersachsen, eine Nordrhein-Westfalen). In jeder Schule wurde die Studie mit jeweils zwei Klassen aller vier Jahrgangsstufen durchgeführt (insg. 24 Klassen), sodass die Stichprobe über die Klassenstufen annähernd gleich verteilt ist $\left(n_{K l l}=101, n_{K l 2}=102, n_{K l 3}=131, n_{K l 4}=122\right)$.

\subsection{Darstellung der entwickelten Testitems}

Die Erfassung des Begriffsverständnisses zu Viereck, Rechteck und Quadrat erfolgte mit Hilfe eines Paper-Pencil Tests. Für jeden Begriff wurden Testitems zu allen drei Indikatoren (Begriffsinhalt, -umfang und -netz) entwickelt. In einer kognitiven Interviewstudie (s.a. Collins 2003) mit $n=4$ Schülerinnen und Schüler pro Klassenstufe und einer Pilotierung mit $N=216$ Schülerinnen und Schülern $\left(n_{K l l}=53, n_{K l 2}=52\right.$, $n_{K 13}=54, n_{K 14}=57$ ) wurden die Items vorab hinsichtlich ihrer Güte überprüft und kritische Items überarbeitet. Nachfolgend werden die Items in der Version dargestellt, die in der vorliegenden Hauptstudie eingesetzt wurde.

Insbesondere für die Operationalisierung des Begriffsumfangs ist aus mathematikdidaktischer Sicht die Unterscheidung der Arten von Eigenschaften hilfreich, um das Begriffsverständnis differenziert(er) erfassen zu können (Heinze 2002b). Aus empirischer Perspektive lassen sich für die Begriffe Viereck, Rechteck und Quadrat entsprechende definierende, nicht-definierende bzw. charakteristische Eigenschaften ableiten (s. Tab. 1). Es liegen bereits Vorschläge für Figurengruppen vor, die 


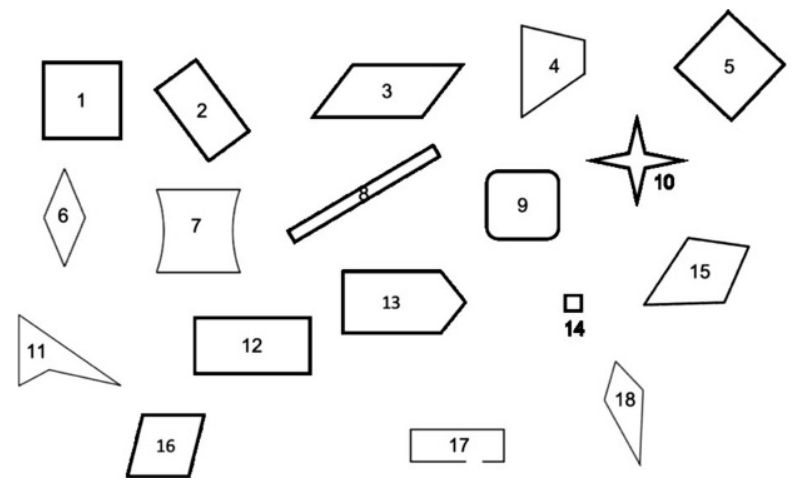

Abb. 2 Figuren zur Aufgabe ,Identifikation von Repräsentanten des Begriffs Viereck/Rechteck/Quadrat ${ }^{\text {}}$ (Begriffsumfang) Anm.: Bezeichnung der Figuren: 1 ,prototypisches Quadrat', 2 ,Rechteck auf der Spitze', 3 ,prototypisches Parallelogramm', 4 , Trapez auf der Spitze‘, 5 ,Quadrat auf der Spitze‘, 6 , langgezogene Raute“, 7 ,gebogene Seiten“, 8 ,schmales Rechteck“, 9 ,abgerundete Ecken“, 10 ,Stern“, 11 ,konkaves Viereck', 12 ,prototypisches Rechteck', 13 ,Fünfeck‘, 14 ,kleines Quadrat', 15 ,unregelmäßige Viereck', 16 ,quadratähnliche Raute', 17 ,offene Seite‘, 18 ,Drachenviereck“

diese definierenden und nicht-definierenden Eigenschaften systematisch variieren (Aktaş Arnas und Aslan 2010; Unterhauser 2020). Basierend auf diesen Vorarbeiten wurde pro Begriff je eine Aufgabe zur Identifikation von Repräsentanten mit jeweils18 Items eingesetzt (s. Abb. 2).

Auswahl und Anordnung der Figuren waren für alle drei Begriffe (Viereck, Rechteck, Quadrat) identisch. Während jedoch bspw. Abb. 2, Figur 16 für den Begriff Viereck einen typischen Repräsentanten darstellt, da die Figur die definierenden sowie die in Tab. 1 gelisteten charakteristischen Eigenschaften aufweist, ist diese Figur ein untypischer Nicht-Repräsentant des Begriffs Quadrat, was durch die Bezeichnung der Figur als ,quadratähnliche Raute' ausgedrückt wird ${ }^{3}$. Die 18 Figuren bilden also für die drei Begriffe unterschiedliche definierende, nicht-definierende bzw. charakteristische Eigenschaften ab (s. Tab. 1), die in den jeweiligen Bezeichnungen der Figuren zum Ausdruck gebracht werden, und stehen somit je nach Begriff exemplarisch für typische bzw. untypische Repräsentanten bzw. Nicht-Repräsentanten (s. Tab. 3). Im Testheft wurden die Figuren auf einer DIN A5-Seite (vgl. Abb. 2) mit der Aufgabenstellung Kreuze alle Vierecke (bzw. Rechtecke bzw. Quadrate) an. dargestellt.

Für das Verständnis des Begriffsinhalts verwendeten bisherige Studien Items zum Definieren bzw. Erklären der Begriffe sowie zur Begründung von Identifikationen (s. Abschn. 5.2). Es lagen jedoch noch keine Items vor, die Eigenschaftswissen über Seiten- und Winkeleigenschaften der Begriffe erfassen. Um Teilaspekte des Begriffsinhalts anhand von Erklärungen, Begründungen und Eigenschaftswissen zu erfassen, wurden für jeden Begriff drei Aufgaben mit insgesamt vier Items zum Begriffsinhalt entwickelt. Im ersten Item wurden die Teilnehmenden aufgefordert, den entsprechenden Begriff einem Kind schriftlich zu erklären. Im zweiten Item

\footnotetext{
3 Eine detaillierte Einordnung aller Figuren bezüglich der definierenden, nicht-definierenden bzw. charakteristischen Eigenschaften findet sich im Anhang.
} 


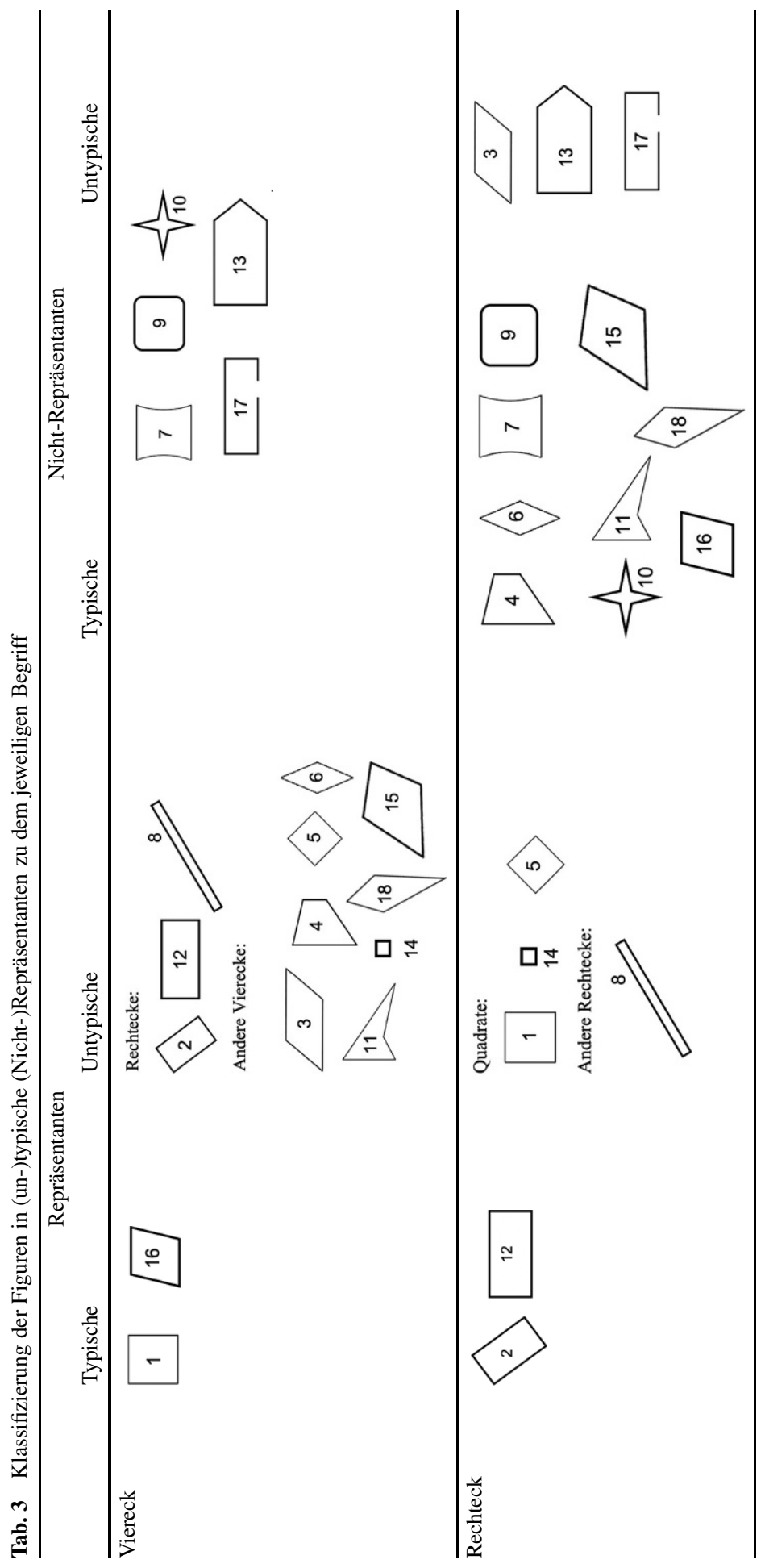




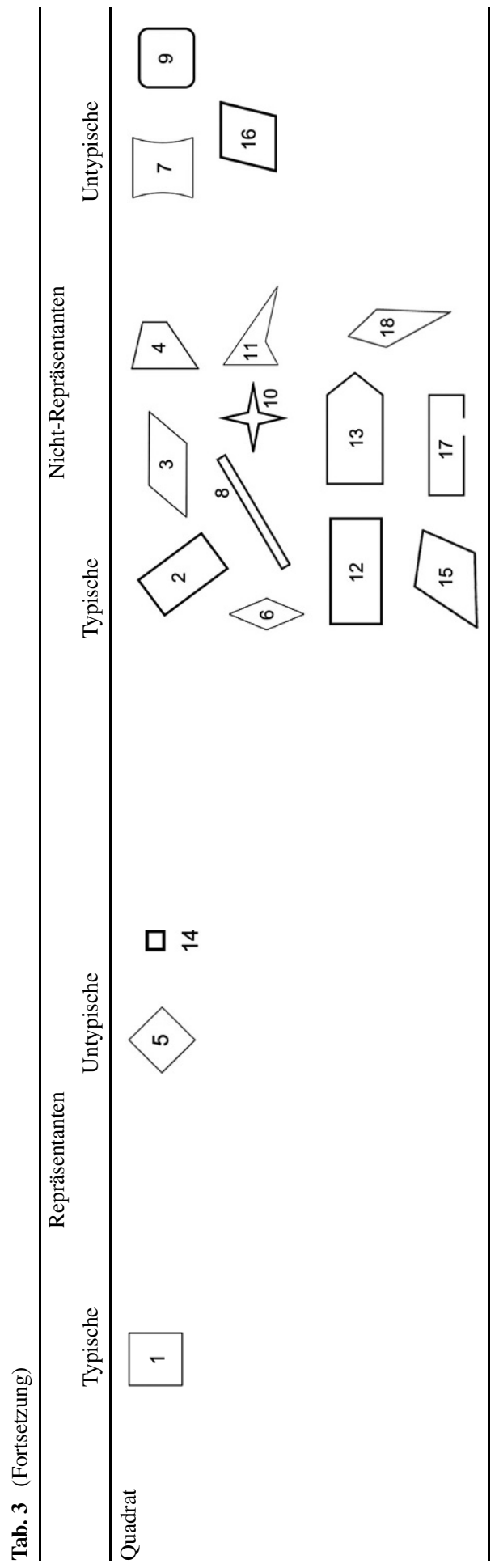


R5: Was stimmt? Kreuze an.

\begin{tabular}{|l} 
Für die Seiten eines Rechtecks gilt: \\
$\square$ Alle Seiten sind gleich lang. \\
$\square$ Alle Seiten sind verschieden lang. \\
$\square$ Je zwei Seiten sind gleich lang. \\
$\square$ Das ist egal. \\
$\square$ Weiß ich nicht.
\end{tabular}

Die Ecken eines Rechtecks sehen so aus:

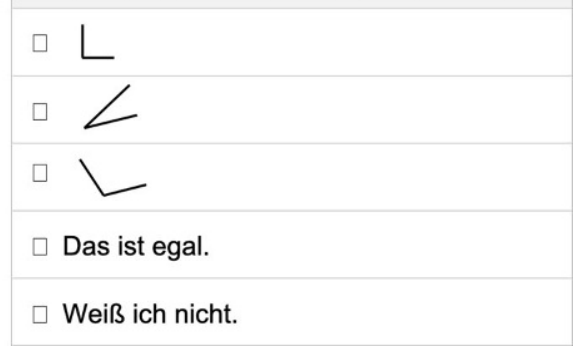

Abb. 3 Beispielitems zur Aufgabe ,Eigenschaften des Begriffs Rechteck‘ (Begriffsinhalt)

galt es, je Begriff eine Figur aus der Aufgabe zum Begriffsumfang (s. Abb. 2) als (Nicht-)Repräsentanten des entsprechenden Begriffs zu identifizieren und die Identifikation schriftlich zu begründen. Aufbauend auf theoretischen Überlegungen zu (nicht-)definierenden Eigenschaften des Begriffs und niedrigen Lösungsquoten der zugehörigen Begriffsumfangitems aus der Pilotierung wurden Figur 11 als untypischer Repräsentant des Begriffs Vierecks, Figur 3 als untypischer Nicht-Repräsentant des Begriffs Rechteck und Figur 14 als untypischer Repräsentant des Begriffs Quadrat ausgewählt. Die dritte Aufgabe überprüfte das Eigenschaftswissen zu den drei Begriffen explizit und bestand aus zwei Multiple-Choice Items pro Begriff zu Winkel- und Seiteneigenschaften (s. Abb. 3).

Zum Begriffsnetz wurden zwei Aufgaben formuliert. In der ersten Aufgabe sollten die Teilnehmenden entscheiden, ob die Figur , prototypisches Rechteck' den Begriff Viereck, Quadrat oder beide Begriffe repräsentiert; in der zweiten Aufgabe, ob die Figur ,prototypisches Quadrat' den Begriff Rechteck, Quadrat oder beide Begriffe repräsentiert.

Tab. 4 gibt einen Überblick über die 68 entwickelten Testitems. Wie dargestellt, wurde das Verständnis der drei Begriffe jeweils mit vergleichbaren Items erfasst, sodass Erkenntnisse hinsichtlich des Begriffsumfangs und -inhalts zwischen den Begriffen vergleichbar sind.

Die Haupterhebung wurde im Frühjahr 2017 von drei geschulten Testleiterinnen im Rahmen ca. einer Unterrichtsstunde durchgeführt. Jede, zu bearbeitende Aufgabe

Tab. 4 Übersicht zum Paper-Pencil Test, BegriV ‘

\begin{tabular}{|c|c|c|c|c|c|}
\hline Begriffsumfang & & Begriffsinhalt & & Begri & \\
\hline $\begin{array}{l}\text { Identifikation } \\
\text { von Reprä- } \\
\text { sentanten zum } \\
\text { Begriff Vier- } \\
\text { eck/Rechteck/ } \\
\text { Quadrat }\end{array}$ & $\begin{array}{l}\text { Erklärung } \\
\text { des Be- } \\
\text { griffs } \\
\text { Viereck/ } \\
\text { Rechteck/ } \\
\text { Quadrat }\end{array}$ & $\begin{array}{l}\text { Begründete Iden- } \\
\text { tifikation von } \\
\text { (Nicht-)Repräsen- } \\
\text { tanten des Begriffs } \\
\text { Viereck/Rechteck/ } \\
\text { Quadrat }\end{array}$ & $\begin{array}{l}\text { Eigenschaften } \\
\text { des Begriffs } \\
\text { Viereck/ } \\
\text { Rechteck/ } \\
\text { Quadrat }\end{array}$ & $\begin{array}{l}\text { Klassenin- } \\
\text { klusion } \\
\text { Viereck } \\
\text { und Recht- } \\
\text { eck }\end{array}$ & $\begin{array}{l}\text { Klassenin- } \\
\text { klusion } \\
\text { Rechteck } \\
\text { und Qua- } \\
\text { drat }\end{array}$ \\
\hline $\begin{array}{l}18 \text { Items pro } \\
\text { Begriff }\end{array}$ & $\begin{array}{l}1 \text { Item pro } \\
\text { Begriff }\end{array}$ & 1 Item pro Begriff & $\begin{array}{l}2 \text { Items pro } \\
\text { Begriff }\end{array}$ & $\begin{array}{l}1 \text { Item pro } \\
\text { Begriffs- } \\
\text { paar }\end{array}$ & $\begin{array}{l}1 \text { Item pro } \\
\text { Begriffs- } \\
\text { paar }\end{array}$ \\
\hline
\end{tabular}


wurde jeweils auf einer Seite im Testheft abgebildet. Zur Bearbeitung der Testaufgaben im Klassenverband wurden die Aufgaben sichtbar an die Wand projiziert sowie durch die Testleitung laut vorgelesen, ehe sie individuell bearbeitet wurden. So konnte für alle Kinder die gleiche Bearbeitungszeit pro Testaufgabe sichergestellt werden.

\subsection{Analyseplan}

Zur Datenauswertung wurden die 18 Items zum Begriffsumfang dichotom (richtig/falsch) codiert. Die Begriffsinhalts-Items der Aufgabe ,Erklärung des Begriffs Viereck/Rechteck/Quadrat' wurden vierstufig codiert: vollständige Definition des Begriffs bzw. Spezifizierung der Eigenschaften (3); Erklärung mit Bezug zu zentralen Eigenschaften (2); prototypische bzw. ganzheitliche Erklärung (1); und falsche Erklärung des Begriffs (0) (s. Tab. 5 im Anhang). Für den Begriff Viereck ist zu beachten, dass mit 2 codierte Antworten bereits vollständige Definitionen präsentieren und Antworten mit dem Code 3 lediglich differenzierter auf die definierenden Eigenschaften des Begriffs Viereck eingehen. Bei den Begriffen Rechteck und Quadrat sind dagegen nur Antworten mit Code 3 vollständige Definitionen. Das Item ,Begründete Identifikation eines (Nicht-)Repräsentanten des Begriffs Viereck/Rechteck/ Quadrat" wurde nur dann als richtig codiert, wenn sich sowohl die Identifikation als auch die Begründung dieser Identifikation als richtig erwies. Würde nur die Identifikation betrachtet werden, wäre nicht gewährleistet, dass in diesem Item Bezug zu den Eigenschaften der Figur hergestellt und der Begriffsinhalt geprüft wird. Die Items zur Aufgabe ,Eigenschaften des Begriffs Viereck/Rechteck/Quadrat' wurden ebenso wie die beiden Items zum Begriffsnetz dichotom codiert.

Da die vorliegende Studie explorativ nach Unterschieden zwischen den Klassenstufen in Bezug auf die Indikatoren des Begriffsverständnisses sucht, werden zunächst deskriptive Statistiken zu allen Items betrachtet. Anschließend wird die Abhängigkeit zwischen den Lösungsquoten jedes Items und der Klassenstufe mittels einfaktorieller Varianzanalysen geprüft (Eid et al. 2015). Post-hoc-Tests erfolgen mittels Bonferroni-Korrektur, da dieser konservative Test sich für eine kleine Anzahl von Gruppen mit unterschiedlicher Stichprobengröße eignet (ebd.).

\section{Ergebnisse}

\subsection{Viereck: Begriffsumfang und -inhalt}

Wird die Lösungshäufigkeit der Items zum Begriffsumfang des Begriffs Viereck deskriptiv getrennt nach der Klassenstufe betrachtet (s. Abb. 4), zeigt sich, dass die typischen Repräsentanten - außer die Figur ,kleines Quadrat' in Klassenstufe 1 sowie die typischen Nicht-Repräsentanten von über $70 \%$ der Lernenden in allen Klassenstufen korrekt identifiziert werden. Auffallend niedrige Lösungsraten in allen Klassenstufen zeigen, mit wenigen Ausnahmen für die Klassenstufen 3 bzw. 4, die untypischen Repräsentanten, insbesondere die Figuren ,konkaves Viereck' und ,Drachenviereck'. 


\begin{tabular}{|c|c|c|c|c|c|c|c|c|c|c|}
\hline \multirow{3}{*}{$\begin{array}{l}\text { Figurengruppe } \\
\text { Figur }\end{array}$} & \multicolumn{8}{|c|}{ Klassenstufe } & & \\
\hline & \multicolumn{2}{|c|}{$\begin{array}{c}1 \\
(n=81)\end{array}$} & \multicolumn{2}{|c|}{$\begin{array}{c}2 \\
(n=102)\end{array}$} & \multicolumn{2}{|c|}{$\begin{array}{c}3 \\
(n=131)\end{array}$} & \multicolumn{2}{|c|}{$\begin{array}{c}4 \\
(n=122)\end{array}$} & \multirow[b]{2}{*}{$\mathbf{F}$} & \multirow[b]{2}{*}{$\eta^{2}$} \\
\hline & $M$ & $S D$ & $M$ & $S D$ & $M$ & $S D$ & $M$ & $S D$ & & \\
\hline \multicolumn{11}{|l|}{ Typische Repräsentanten } \\
\hline 1 ,prototypisches Quadrat & ,96 & 0,19 & ,98 & 0,14 & 99 & 0,12 & 1,00 & 0,00 & 1,446 & - \\
\hline 16 ,quadratähnliche Raute & ,78 & 0,42 & ,86 & 0,35 & 89 & 0,32 &, 82 & 0,39 & 1,723 & - \\
\hline 5 ,Quadrat auf der Spitze" & ,77 & 0,43 & 89 & 0,31 & ,97 & 0,17 & ,95 & 0,22 & $10,137^{* *}$ &, 07 \\
\hline 14, kleines Quadrat & ,59 & 0,49 & ,74 & 0,44 & ,86 & 0,35 & 89 & 0,32 & $11,247^{* *}$ &, 07 \\
\hline \multicolumn{11}{|l|}{ Untypische Repräsentanten } \\
\hline 12 ,prototypisches Rechteck & ,20 & 0,40 &, 18 & 0,38 &, 39 & 0,49 & ,47 & 0,50 & $10,618^{* *}$ &, 07 \\
\hline 3 ,prototypisches Parallelogramm &, 15 & 0,36 & ,19 & 0,39 & ,44 & 0,50 & ,40 & 0,49 & $11,148^{* *}$ &, 07 \\
\hline 2, Rechteck auf der Spitze“ & ,16 & 0,37 & ,19 & 0,39 & 37 & 0,48 & ,45 & 0,50 & $10,276^{* *}$ &, 07 \\
\hline 8 , schmales Rechteck' & ,09 & 0,28 & ,13 & 0,34 & 31 & 0,46 &, 38 & 0,49 & $11,743^{* *}$ &, 08 \\
\hline 15 ,unregelmäßiges Viereck &, 21 & 0,41 &, 27 & 0,44 &, 52 & 0,50 & ,42 & 0,50 & $9,625^{* *}$ & ,06 \\
\hline 4 ,Trapez auf der Spitze &, 11 & 0,32 &, 12 & 0,32 & ,38 & 0,49 &, 33 & 0,47 & $11,973^{* *}$ &, 08 \\
\hline 6 , langgezogene Raute“ &, 10 & 0,30 & ,18 & 0,38 &, 35 & 0,48 & ,36 & 0,48 & $9,136^{* *}$ &, 06 \\
\hline 18, Drachenviereck & ,07 & 0,26 &, 14 & 0,35 & 31 & 0,46 & 26 & 0,44 & $7,427^{* *}$ &, 05 \\
\hline 11 ,konkaves Viereck' & 01 & 0,11 & ,08 & 0,27 & 24 & 0,43 & 20 & 0,40 & $9,270^{* *}$ &, 06 \\
\hline \multicolumn{11}{|l|}{ Untypische Nicht-Repräsentanten } \\
\hline 7 ,gebogene Seiten & ,47 & 0,50 & ,48 & 0,50 & 39 & 0,49 & ,48 & 0,50 & 0,920 & - \\
\hline 9 , abgerundete Ecken &, 19 & 0,39 & ,33 & 0,47 &, 42 & 0,50 &, 56 & 0,50 & $10,776^{* *}$ & 07 \\
\hline \multicolumn{11}{|l|}{ Typische Nicht-Repräsentanten } \\
\hline 10, Stern & ,93 & 0,26 & ,95 & 0,22 & ,92 & 0,27 & ,93 & 0,26 & 0,272 & - \\
\hline 13 ,Fünfeck & ,99 & 0,11 & ,97 & 0,17 & 1,00 & 0,00 & 1,00 & 0,00 & 2,383 & - \\
\hline 17 ,offene Seite &, 85 & 0,36 & ,86 & 0,35 &, 72 & 0,45 & ,79 & 0,41 & $3,163^{*}$ &, 02 \\
\hline
\end{tabular}

Abb. 4 Lösungshäufigkeit der Items zum Begriffsumfang zum Begriff Viereck getrennt nach Klassenstufe. Anmerkung: hellgraue Schattierung markiert Lösungsquoten über $75 \%$, dunkelgraue Schattierung markiert Lösungsquoten unter $40 \%$. Effektstärken werden nach Cohen (1988) wie folgt eingeteilt: $\eta^{2} \approx 0,01$ : kleiner Effekt; $\eta^{2} \approx 0,06$ : mittlerer Effekt; $\eta^{2} \approx 0,14$ : großer Effekt

Signifikante Unterschiede zwischen den Klassenstufen zeigen sich bei den Lösungsraten aller Figuren außer den typischen Repräsentanten ,prototypisches Quadrat' und ,quadratähnliche Raute', dem untypischen Nicht-Repräsentant ,gebogene Seiten ' sowie den typischen Nicht-Repräsentanten ,Stern', und ,Fünfeck' (s. Abb. 4). Bei den Figuren ,offene Seite ' und ,Drachenviereck' zeigt sich ein kleiner Effekt der Klassenstufe. Bei den übrigen Figuren mit einem signifikanten Unterschied der Lösungsraten zwischen den Klassenstufen ergibt sich ein mittlerer Effekt.

Post-Hoc-Tests zeigen, dass der typische Repräsentant ,Quadrat auf der Spitze in den Klassenstufen 2 und 3 als Gruppe signifikant besser identifiziert wird als in der Klassenstufe 1. Für den typischen Repräsentanten ,kleines Quadrat‘ zeigt sich ein signifikanter Unterschied in den Lösungsraten zwischen der Klassenstufe 1 und den Klassenstufen 3 und 4 sowie zwischen der Klassenstufe 2 und 4 - mit einem besseren Abschneiden der jeweils höheren Jahrgangsstufe. Dies deutet darauf hin, dass diese beiden Figuren für einige Kinder erst im Laufe der Grundschulzeit zu typischen Repräsentanten des Begriffs Viereck werden.

In Bezug auf die Identifikationen der untypischen Repräsentanten des Begriffs können für alle Figuren dieser Gruppe - bis auf die Figuren , unregelmäßiges Vier- 
eck' und ,Drachenviereck' - die Klassenstufen 1 und 2 sowie die Klassenstufen 3 und $4 \mathrm{zu}$ einer Gruppe zusammengefasst werden, die sich jeweils signifikant voneinander unterscheiden, wobei die Klassenstufen 3/4 tendenziell besser abschneiden als die Klassenstufen 1/2. Diese Ergebnisse deuten darauf hin, dass diese untypischen Repräsentanten des Begriffs Viereck im Laufe der Grundschulzeit von immer mehr Kindern als Vierecke identifiziert werden - insbesondere, wenn sie typische Repräsentanten anderer Vierecksfiguren (bspw. Parallelogramm) sind. Bei den untypischen Repräsentanten ,unregelmäßiges Viereck' und ,Drachenviereck' zeigen Post-Hoc-Tests einen signifikanten Unterschied zwischen der Klassenstufe 1 und den Klassenstufen 3 und 4 sowie zwischen der Klassenstufe 2 und 3. Bei beiden Figuren werden jeweils in der dritten Klassenstufe die meisten korrekten Identifikationen getroffen und dabei zeigen sich innerhalb der Figurengruppe untypische Repräsentanten für die Figur ,unregelmäßige Viereck' in Klassenstufe 3 die häufigsten korrekten Identifikationen.

Für den untypischen Nicht-Repräsentanten ,abgerundete Ecken“ zeigen Post-HocTests einen signifikanten Unterschied in den Lösungsraten zwischen der Klassenstufe 1 und den Klassenstufen 3 und 4 sowie zwischen der Klassenstufe 2 und 4 mit einem besseren Abschneiden der jeweils höheren Jahrgangsstufe. Auch für diese Figur werden im Laufe der Grundschulzeit mehr korrekte Identifikationsentscheidungen getroffen.

Bei dem typischen Nicht-Repräsentanten ,offene Seite' zeigen Post-Hoc-Tests einen signifikanten Unterschied zwischen der Klassenstufe 2 und 3. Einzig bei dieser Figur zeigt sich tendenziell eine Abnahme an korrekten Identifikationsentscheidungen im Laufe der Grundschulzeit.

Zusammenfassend deuten die Ergebnisse zum Begriffsumfang des Begriffs Viereck daraufhin, dass typische Repräsentanten und Nicht-Repräsentanten bereits zu Beginn der Grundschulzeit sicher korrekt identifiziert werden - spätestens jedoch ab Klasse 3. Die untypischen Repräsentanten und Nicht-Repräsentanten werden dagegen über alle vier Klassenstufen - mit der Ausnahme weniger Figuren - selten korrekt identifiziert. Tendenziell erhöht sich jedoch der Anteil an korrekten Identifikationen im Laufe der Grundschulzeit. Für die typischen Nicht-Repräsentanten zeigt sich - ähnlich wie für die typischen Repräsentanten - keine Weiterentwicklung im Laufe der Grundschulzeit, jedoch bei korrekten Identifikationsentscheidungen auf mittlerem Niveau. Für die Figur ,offene Seite“ zeigt sich sogar eine Abnahme der korrekten Identifikationen im Vergleich der Klassenstufen.

Bezüglich des Begriffsinhalts zum Begriff Viereck zeigt das Item zur Erklärung des Begriffs Viereck auf deskriptiver Ebene, dass der Mittelwert der Klassenstufe $1(M=1,51, S D=0,85)$ höher ausfällt als die Mittelwerte der Klassenstufen 2 $(M=1,46, S D=0,92), 3(M=1,04, S D=0,76)$ und $4(M=1,32, S D=0,94)$ und der Anteil der ganzheitlichen Erklärungen bzw. der Erklärungen, die den Begriff Viereck auf den Prototyp Quadrat reduzieren, in der Klassenstufe 3 am höchsten liegt (s. Abb. 5).

Die Varianzanalyse deckt einen signifikanten, aber schwachen Effekt der Klassenstufe $\left(F(3,421)=6,336, p<0,001, \eta^{2}=0,04\right)$ auf. Post-Hoc-Tests zeigen einen signifikanten Unterschied zwischen den Klassenstufen 2 und 3. 


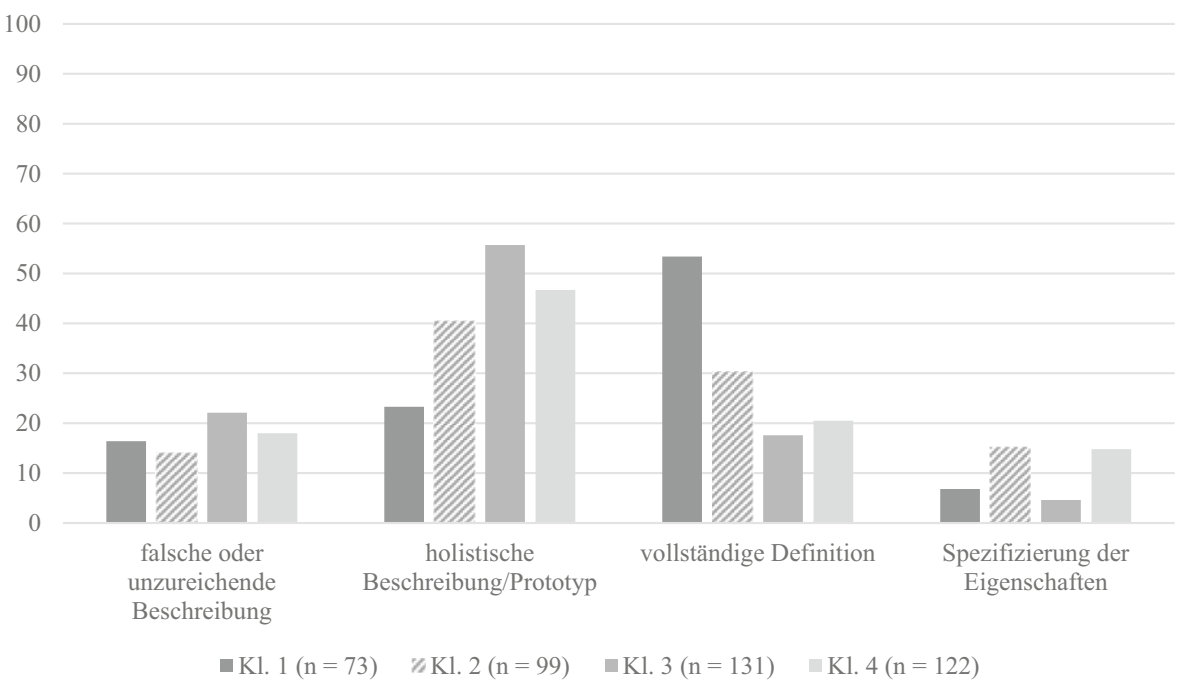

Abb. 5 Erklärungen des Begriffs Viereck nach Klassenstufe getrennt, Angaben in \%

Bezüglich der Lösungshäufigkeit des Items zur begründeten Identifikation der Figur ,konkaves Viereck' als Repräsentant des Begriffs Viereck zeigt sich ebenfalls ein signifikanter Unterschied zwischen den Klassenstufen $(F(3,415)=21,421$, $p<0,001)$ mit einem mittleren Effekt $\left(\eta^{2}=0,13\right)$. Post-Hoc-Tests ergeben, dass sich die Klassenstufen $1(n=69, M=0,04, S D=0,21)$ und $2(n=98, M=0,07, S D=0,26)$ sowie die Klassenstufen $3(n=130, M=0,42, S D=0,50)$ und $4(n=122, M=0,31$, $S D=0,47)$ jeweils zu einer Gruppe zusammenfassen lassen, die sich signifikant voneinander unterscheiden.

Hinsichtlich der Lösungshäufigkeiten der Items zu den Seiten- und Winkeleigenschaften des Vierecks zeigt sich ein signifikanter mittlerer Effekt der Klassenstufe (Seite: $F(3,428)=10,289, p<0,001, \eta^{2}=0,07$; Winkel: $F(3,431)=9,350$, $\left.p<0,001, \eta^{2}=0,06\right)$. Für beide Items zeigen Post-Hoc-Tests paarweise Unterschiede zwischen den Klassenstufen 2 (Seite: $n=100, M=0,01, S D=0,10$; Winkel: $n=102, M=0,03, S D=0,20), 3$ (Seite: $n=130, M=0,19, S D=0,40$; Winkel: $n=129$, $M=0,23, S D=0,42$ ) und 4 (Seite: $n=122, M=0,25, S D=0,43$; Winkel: $n=122$, $M=0,25, S D=0,43)$.

\subsection{Rechteck: Begriffsumfang und -inhalt}

Wird die Lösungshäufigkeit der Items zum Begriffsumfang des Begriffs Rechteck deskriptiv getrennt nach Klassenstufen betrachtet (s. Abb. 6), zeigt sich, dass der typische Repräsentant - außer in Klassenstufe 2 - und die typischen Nicht-Repräsentanten - außer die Figur ,abgerundete Ecken“ in Klassenstufe 1 - von über $90 \%$ der Schülerinnen und Schüler aller Klassenstufen korrekt identifiziert werden. Auffallend niedrige Lösungsraten in allen Klassenstufen zeigen die untypischen Repräsentanten ,prototypisches Quadrat', ,Quadrat auf der Spitze“ und ,kleines Quadrat‘. 


\begin{tabular}{|c|c|c|c|c|c|c|c|c|c|c|}
\hline \multirow{3}{*}{$\begin{array}{l}\text { Figurengruppe } \\
\text { Figur }\end{array}$} & \multicolumn{8}{|c|}{ Klassenstufe } & & \\
\hline & \multicolumn{2}{|c|}{$\begin{array}{c}1 \\
(n=81)\end{array}$} & \multicolumn{2}{|c|}{$\begin{array}{c}2 \\
(n=102)\end{array}$} & \multicolumn{2}{|c|}{$\left.\begin{array}{c}3 \\
(n=131)\end{array}\right)$} & \multicolumn{2}{|c|}{$\begin{array}{c}4 \\
(n=122)\end{array}$} & \multirow[b]{2}{*}{$\mathbf{F}$} & \multirow[b]{2}{*}{$\eta^{2}$} \\
\hline & $M$ & $S D$ & $M$ & $S D$ & $M$ & $S D$ & $M$ & $S D$ & & \\
\hline \multicolumn{11}{|l|}{ Typische Repräsentanten } \\
\hline 12 ,prototypisches Rechteck' & ,90 &, 30 &, 87 &, 34 & ,93 &, 25 & ,93 & ,26 & 0,983 & - \\
\hline \multicolumn{11}{|l|}{ Untypische Repräsentanten } \\
\hline 2, Rechteck auf der Spitze &, 77 & ,43 &, 81 &, 39 & ,92 & ,28 &, 88 &, 33 & $3,704^{*}$ &, 03 \\
\hline 8, schmales Rechteck' &, 52 &, 50 &, 53 &, 50 & 65 &, 48 &, 70 & ,46 & $3,762^{*}$ &, 03 \\
\hline \multicolumn{11}{|l|}{ Untypische Repräsentanten (Quadrate) } \\
\hline 1 ,prototypisches Quadrat' & ,09 &, 28 &, 04 & ,20 &, 03 &, 17 &, 08 &, 28 & 1,670 & - \\
\hline 5 ,Quadrat auf der Spitze &, 10 &, 30 &, 04 &, 20 &, 04 &, 19 &, 10 &, 30 & 2,101 & - \\
\hline 14 ,kleines Quadrat" & ,05 &, 22 &, 02 &, 14 & ,02 &, 15 & ,06 &, 23 & 1,129 & - \\
\hline \multicolumn{11}{|l|}{ Untypische Nicht-Repräsentanten } \\
\hline 3 ,prototypisches Parallelogramm &, 59 &, 49 &, 44 &, 50 &, 34 & ,48 &, 48 &, 50 & $4,506^{*}$ &, 03 \\
\hline 13 ,Fünfeck &, 89 &, 32 &, 87 &, 34 & ,92 &, 28 & ,93 &, 25 & 0,974 & - \\
\hline 17 , offene Seite‘ &, 22 & ,42 &, 22 &, 41 &, 26 &, 44 &, 48 &, 50 & $9,051^{* *}$ &, 06 \\
\hline \multicolumn{11}{|l|}{ Typische Nicht-Repräsentanten } \\
\hline 16, quadratähnliche Raute & ,94 &, 24 & ,97 &, 17 & ,97 &, 17 & ,94 &, 23 & 0,738 & - \\
\hline 15 , unregelmäßiges Viereck & ,96 &, 19 &, 97 &, 17 & ,98 &, 12 & ,97 &, 18 & 0,380 & - \\
\hline 4 ,Trapez auf der Spitze“ & ,96 &, 19 & ,99 &, 10 & ,98 &, 12 & ,98 &, 13 & 0,690 & - \\
\hline 6 , langgezogene Raute & ,96 &, 19 & ,98 &, 14 & ,98 &, 15 & ,98 &, 13 & 0,332 & - \\
\hline 18 ,Drachenviereck & ,98 &, 16 & ,98 &, 14 & ,99 &, 09 & ,98 &, 16 & 0,427 & - \\
\hline 11 ,konkaves Viereck' & ,99 &, 11 & ,98 &, 14 & ,98 &, 12 & 1,00 &, 00 & 0,719 & - \\
\hline 10, Stern' & ,99 &, 11 & ,98 &, 14 & 1,00 &, 00 & 1,00 &, 00 & 1,510 & - \\
\hline 7 ,gebogene Seiten & ,94 &, 24 & ,96 &, 20 & ,96 &, 19 & ,96 &, 20 & 0,264 & - \\
\hline 9 , abgerundete Ecken &, 88 &, 33 & ,96 &, 20 &, 97 &, 17 & ,98 &, 16 & $4,272^{*}$ &, 03 \\
\hline
\end{tabular}

Abb. 6 Lösungshäufigkeit der Items zum Begriffsumfang zum Begriff Rechteck getrennt nach Klassenstufe. Anmerkung: hellgraue Schattierung markiert Lösungsquoten über $75 \%$, dunkelgraue Schattierung markiert Lösungsquoten unter $40 \%$. Effektstärken werden nach Cohen (1988) wie folgt eingeteilt: $\eta^{2} \approx 0,01$ : kleiner Effekt; $\eta^{2} \approx 0,06$ : mittlerer Effekt; $\eta^{2} \approx 0,14$ : großer Effekt

Signifikante Unterschiede zwischen den Klassenstufen zeigen sich bei den Lösungshäufigkeiten der untypischen Repräsentanten, die keine quadratischen Rechtecke sind (,Rechteck auf der Spitze', ,schmales Rechteck'), mit jeweils kleinem Effekt sowie den untypischen Nicht-Repräsentanten ,prototypisches Parallelogramm` und ,abgerundete Ecken', mit jeweils kleinem Effekt und ,offene Seite' mit mittlerem Effekt (s. Abb. 6).

Für den untypischen Repräsentanten ,Rechteck auf der Spitze‘ zeigen Post-HocTests, dass ein signifikanter Unterschied zwischen Lösungsraten der Klassenstufe 2 und 3 besteht. Dabei wird die Figur in der Klassenstufe 3 am häufigsten korrekt als Repräsentant des Begriffs Rechteck identifiziert. Für die Figur ,schmales Rechteck ‘ lassen sich in den Post-Hoc Tests keine Gruppenunterschiede ausmachen.

Bei dem untypischen Nicht-Repräsentanten, prototypisches Parallelogramm`zeigen Post-Hoc-Tests, dass ein signifikanter Unterschied zwischen der Klassenstufe 2 und 3 vorliegt. Dabei wird die Figur in der Klassenstufe 2 häufiger korrekt als NichtRepräsentant identifiziert. Bei dem untypischen Nicht-Repräsentanten ,offene Seite 
lassen sich dagegen die Klassenstufen 1 bis 3 zu einer Gruppe zusammenfassen, die sich signifikant von der Klassenstufe 4 unterscheidet. Die Lernenden der Klassenstufe 4 wählen diese Figur seltener als Repräsentant des Begriffs Rechteck aus als die Lernenden der übrigen Klassen.

Bei dem typischen Nicht-Repräsentanten ,abgerundete Ecken' können die Klassenstufen 2, 3 und 4 hinsichtlich ihrer Lösungshäufigkeit zusammengefasst werden, die sich als Gruppe signifikant von Klassenstufe 1 unterscheidet und die Figur seltener als Repräsentant auswählt.

Zusammenfassend zeigen die Ergebnisse zum Begriffsumfang des Begriff Rechteck, dass typische Repräsentanten und Nicht-Repräsentanten ab Klassenstufe 1 sicher korrekt identifiziert werden. Gleiches gilt auch für den bislang als untypisch beschriebenen Repräsentanten ,Rechteck auf der Spitze' (Variation des typischen Repräsentanten in der Orientierung parallel zum Blattrand) und Nicht-Repräsentanten ,Fünfeck' (Ergänzung des typischen Repräsentanten um eine vorspringende Ecke). Aus der Figurengruppe der untypischen Repräsentanten zeigen insbesondere die quadratischen Rechtecke niedrige Lösungsquoten, ohne dass sich Entwicklungstendenzen zwischen den Klassenstufen erkennen lassen. Aus der Figurengruppe der untypischen Nicht-Repräsentanten weist insbesondere die Figur ,offene Seite ' niedrige Lösungsquoten auf, jedoch erhöht sich diese Lösungsquote mit zunehmender Klassenstufe.

Zum Begriffsinhalt des Begriffs Rechteck zeigen sich hinsichtlich der Erklärung des Begriffs signifikante Unterschiede zwischen den Klassenstufen $(F(3,394)=$ $15,221, p<0,001)$ mit mittlerem Effekt $\left(\eta^{2}=0,10\right)$. Post-Hoc-Tests zeigen, dass sich die Klassenstufen $1(M=0,50, S D=0,57)$ und $2(M=0,53, S D=0,60)$ sowie $3(M=0,89, S D=0,70)$ und $4(M=1,09, S D=0,87)$ hinsichtlich der Erklärungen des Begriffs Rechteck jeweils zu einer Gruppe zusammenfassen lassen, zwischen denen ein signifikanter Unterschied besteht. Auf deskriptiver Ebene fällt zusätzlich auf, dass über $40 \%$ der Erklärungen in allen Klassenstufen ganzheitlich bzw. prototypisch sind (s. Abb. 7).

Bezüglich des Items zur begründeten Identifikation der Figur ,prototypisches Parallelogramm ‘ als Nicht-Repräsentant des Begriffs Rechteck zeigt sich kein signifikanter Unterschied zwischen den Klassenstufen $(F(3,381)=1,762, p=0,154)$. Dabei lösen 33,85\% der Lernenden in Klasse $1(n=65, S D=0,48), 23,16 \%$ der Lernenden in Klasse $2(n=95, S D=0,42), 30,40 \%$ der Lernenden in Klasse $3(n=125$, $S D=0,46)$ und $38,00 \%$ der Lernenden in Klasse $4(n=100, S D=0,49)$ das Item korrekt. Auch in Bezug auf die Lösungsquoten der Items zu den Winkeleigenschaften zeigen sich keine signifikanten Unterschiede zwischen den Klassenstufen $(F(3,305)=0,072, p=0,552)$. Im Mittel wählen 18,67\% der Lernenden in Klasse 1 $(n=75, S D=0,39), 8,91 \%$ der Lernenden in Klasse $2(n=101, S D=0,29), 5,61 \%$ der Lernenden in Klasse $3(n=107, S D=0,23)$ und 4,92\% der Lernenden in Klasse 4 ( $n=122, S D=0,22)$ die korrekte Lösung aus. Hinsichtlich der Seiteneigenschaften zeigt sich dagegen ein signifikanter Unterschied $(F(3,393)=5,857, p=0,001)$ mit kleinem Effekt $\left(\eta^{2}=0,04\right)$. Post-Hoc Test zeigen einen Unterschied zwischen der Klassenstufe $1(n=75, M=0,13, S D=0,34)$ und $3(n=104, M=0,33, S D=0,47)$ sowie zwischen den Klassenstufen 3 und $4(n=120, M=0,13, S D=0,33)$. 


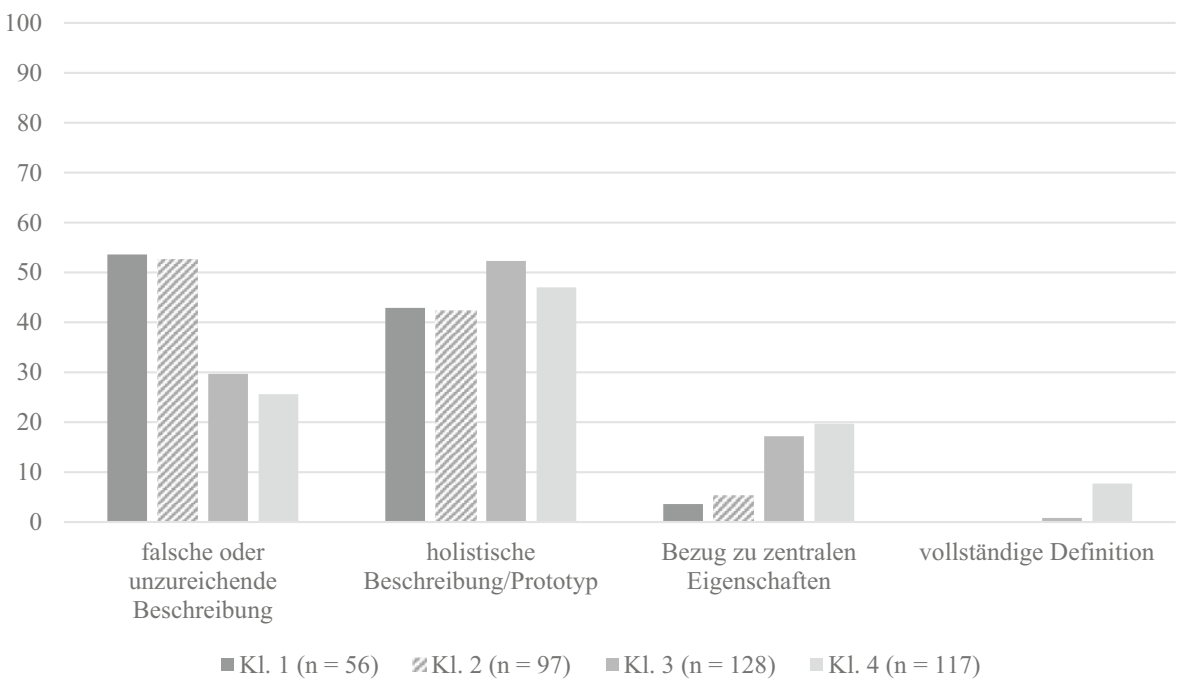

Abb. 7 Erklärungen des Begriffs Rechteck nach Klassenstufe getrennt, Angaben in \%

\subsection{Quadrat: Begriffsumfang und -inhalt}

Bezüglich des Begriffsumfangs des Begriffs Quadrat zeigt die deskriptive Betrachtung der Lösungshäufigkeit aller Items getrennt nach der Klassenstufe (Abb. 8), dass typische Nicht-Repräsentanten - außer die Figuren ,prototypisches Rechteck " und ,Rechteck auf der Spitze‘ in Klassenstufe 4 - von über $90 \%$ der Lernenden aller Klassenstufen korrekt identifiziert werden. Niedrige Lösungsraten in Klassenstufe 1 und 2 zeigt der untypische Repräsentant ,kleines Quadrat‘. Der typische Repräsentant ,prototypisches Quadrat' zeigt mit Lösungsquoten im mittleren Bereich im Vergleich zu den typischen Repräsentanten der Begriffe Rechteck und Viereck ebenfalls wenig korrekte Identifikationen in allen Klassenstufen.

Signifikante Unterschiede zwischen den Klassenstufen zeigen sich bezüglich aller typischen und untypischen Repräsentanten des Begriffs Quadrat, bei allen untypischen Nicht-Repräsentanten sowie allen typischen Nicht-Repräsentanten mit rechten Winkeln und bei den weiteren typischen Nicht-Repräsentanten , unregelmäßiges Viereck', ,Trapez auf der Spitze‘ und ,Drachenviereck‘ (s. Abb. 8).

Hinsichtlich des typischen Repräsentanten ,prototypisches Quadrat' können die Klassenstufen 1 und 2 zu einer Gruppe zusammengefasst werden, die sich signifikant von der Klassenstufe 3 unterscheidet - dabei schneiden die Lernenden in höheren Klassenstufen besser ab.

Für den untypischen Repräsentanten, Quadrat auf der Spitze ' unterscheidet sich Klassenstufe 1 signifikant von Klassenstufe 3. Für den untypischen Repräsentanten ,kleines Quadrat' unterscheidet sich die Klassenstufe 1 sowohl von Klassenstufe 3 als auch 4 signifikant und zudem die Klassenstufe 2 signifikant von Klassenstufe 3. Bei beiden Figuren zeigt sich in Klassenstufe 3 die höchste Lösungshäufigkeit.

Bei dem untypischen Nicht-Repräsentanten ,abgerundete Ecken' lassen sich die Klassenstufen 1 und 2 zu einer Gruppe zusammenfassen, die sich signifikant von den 


\begin{tabular}{|c|c|c|c|c|c|c|c|c|c|c|}
\hline \multirow{3}{*}{$\begin{array}{l}\text { Figurengruppe } \\
\text { Figur }\end{array}$} & \multicolumn{8}{|c|}{ Klassenstufe } & & \\
\hline & \multicolumn{2}{|c|}{$\begin{array}{c}1 \\
(n=81)\end{array}$} & \multicolumn{2}{|c|}{$\begin{array}{c}2 \\
(n=102)\end{array}$} & \multicolumn{2}{|c|}{$\begin{array}{c}3 \\
(n=131)\end{array}$} & \multicolumn{2}{|c|}{$\begin{array}{c}4 \\
(n=122)\end{array}$} & \multirow[b]{2}{*}{$\mathbf{F}$} & \multirow[b]{2}{*}{$\eta^{2}$} \\
\hline & $M$ & $S D$ & $M$ & $S D$ & $M$ & $S D$ & $M$ & $S D$ & & \\
\hline \multicolumn{11}{|l|}{ Typische Repräsentanten } \\
\hline 1 ,prototypisches Quadrat" & ,47 &, 50 &, 50 &, 50 & ,70 & ,46 & ,61 & ,49 & $5,191^{*}$ & 03 \\
\hline \multicolumn{11}{|l|}{ Untypische Repräsentanten } \\
\hline 5,Quadrat auf der Spitze“ & ,43 &, 50 &, 56 &, 50 &, 71 & ,46 &, 58 &, 50 & $5,675^{*}$ & 04 \\
\hline 14 ,kleines Quadrat &, 26 & ,44 & ,38 & ,49 &, 56 &, 50 & ,45 &, 50 & $6,718^{* *}$ &, 04 \\
\hline \multicolumn{11}{|l|}{ Untypische Nicht-Repräsentanten } \\
\hline 16 ,quadratähnliche Raute“ & ,67 &, 47 &, 51 &, 50 & ,35 & ,48 & ,68 & ,47 & $12,208^{* *}$ &, 08 \\
\hline 7 , gebogene Seiten' & ,78 & ,42 & ,73 & ,45 & ,66 & ,47 & ,91 & ,29 & $7,949^{* *}$ &, 05 \\
\hline 9 , abgerundete Ecken" & ,63 & ,49 & ,63 & ,49 & ,44 &, 50 &, 81 & ,39 & $13,207^{* *}$ & ,08 \\
\hline \multicolumn{11}{|c|}{ Typische Nicht-Repräsentanten (mit rechten Winkeln) } \\
\hline 12 ,prototypisches Rechteck' & ,89 & ,32 &, 86 & ,35 &, 86 & ,35 & 68 & 47 & $7,377^{* *}$ &, 05 \\
\hline 2 , Rechteck auf der Spitze“ &, 86 &, 34 &, 85 & ,36 &, 84 & ,37 &, 70 & ,46 & $4,164^{*}$ & 03 \\
\hline 8, schmales Rechteck' & ,95 &, 22 & ,92 &, 27 & ,92 &, 27 & 80 & ,41 & $5,879^{*}$ &, 04 \\
\hline \multicolumn{11}{|l|}{ Typische Nicht-Repräsentanten } \\
\hline $\begin{array}{l}3 \text {,prototypisches Parallelo- } \\
\text { gramm }\end{array}$ &, 86 & ,34 &, 82 & ,38 & ,82 & ,39 & ,79 & ,41 & 0,653 & - \\
\hline 15 ,unregelmäßiges Viereck‘ & 89 &, 32 & 80 & ,40 & ,76 & ,43 & ,92 & ,28 & $4,651^{*}$ & 03 \\
\hline 4, ,Trapez auf der Spitze“ & ,96 &, 19 & ,93 & 25 & ,86 & ,35 & ,97 & ,18 & $4,783^{*}$ &, 03 \\
\hline 6 , langgezogene Raute & ,89 &, 32 &, 84 &, 37 &, 87 & ,34 & ,90 &, 30 & 0,636 & - \\
\hline 18, Drachenviereck & ,93 & ,26 & 85 & ,36 & ,93 & 25 & ,98 &, 13 & $4,781^{*}$ & 03 \\
\hline 11 ,konkaves Viereck & ,98 &, 16 & ,97 &, 17 & ,95 & ,23 & ,99 & ,09 & 1,526 & - \\
\hline 10 ,Stern' & ,95 &, 22 & ,97 &, 17 & ,98 &, 12 & ,98 &, 13 & 0,968 & - \\
\hline 13 ,Fünfeck' & ,98 &, 16 & ,99 &, 10 & ,97 &, 17 & ,97 &, 18 & 0,471 & - \\
\hline 17 ,offene Seite &, 86 & ,34 &, 89 &, 31 &, 88 & ,33 &, 89 &, 32 & 0,123 & - \\
\hline
\end{tabular}

Abb. 8 Lösungshäufigkeit der Items zum Begriffsumfang zum Begriff Quadrat getrennt nach Klassenstufe. Anmerkung: hellgraue Schattierung markiert Lösungsquoten über $75 \%$, dunkelgraue Schattierung markiert Lösungsquoten unter $40 \%$. Effektstärken werden nach Cohen (1988) wie folgt eingeteilt: $\eta^{2} \approx 0,01$ : kleiner Effekt; $\eta^{2} \approx 0,06$ : mittlerer Effekt; $\eta^{2} \approx 0,14$ : großer Effekt

Klassenstufen 3 (schneidet schlechter ab als Klasse 1/2) sowie 4 (schneidet besser ab als Klasse 1/2) unterscheidet. Zudem zeigen sich signifikante Unterschiede zwischen den Klassenstufen 3 und 4. Für die Figuren ,gebogene Seiten“ und ,quadratähnliche Raute ' lassen sich die Klassenstufen 2 und 3 zu einer Gruppe zusammenfassen, die sich signifikant von der Klassenstufe 4 unterscheidet, wobei jeweils die Klassenstufe 4 besser abschneidet. Für die Figur ,quadratähnliche Raute' zeigt sich darüber hinaus ein signifikanter Unterschied zwischen der Klassenstufe 1 und 3, wobei die Figur häufiger in Klassenstufe 1 als Nicht-Repräsentant identifiziert wird.

Bei den typischen Nicht-Repräsentanten mit rechten Winkeln zeigen Post-HocTests, dass die Klassenstufen 1, 2 und 3 hinsichtlich ihrer Lösungshäufigkeit zusammengefasst werden können und sich signifikant von der Klassenstufe 4 unterscheiden. Dabei werden alle drei Figuren in Klassenstufe 4 seltener als Repräsentant identifiziert. Sowohl bezüglich der Figur , unregelmäßiges Viereck' als auch ,Trapez auf der Spitze` zeigen sich signifikante Unterschiede zwischen den Klassenstufen 1 und 3, wobei die Figuren in der Klassenstufe 3 seltener korrekt als Nicht-Reprä- 


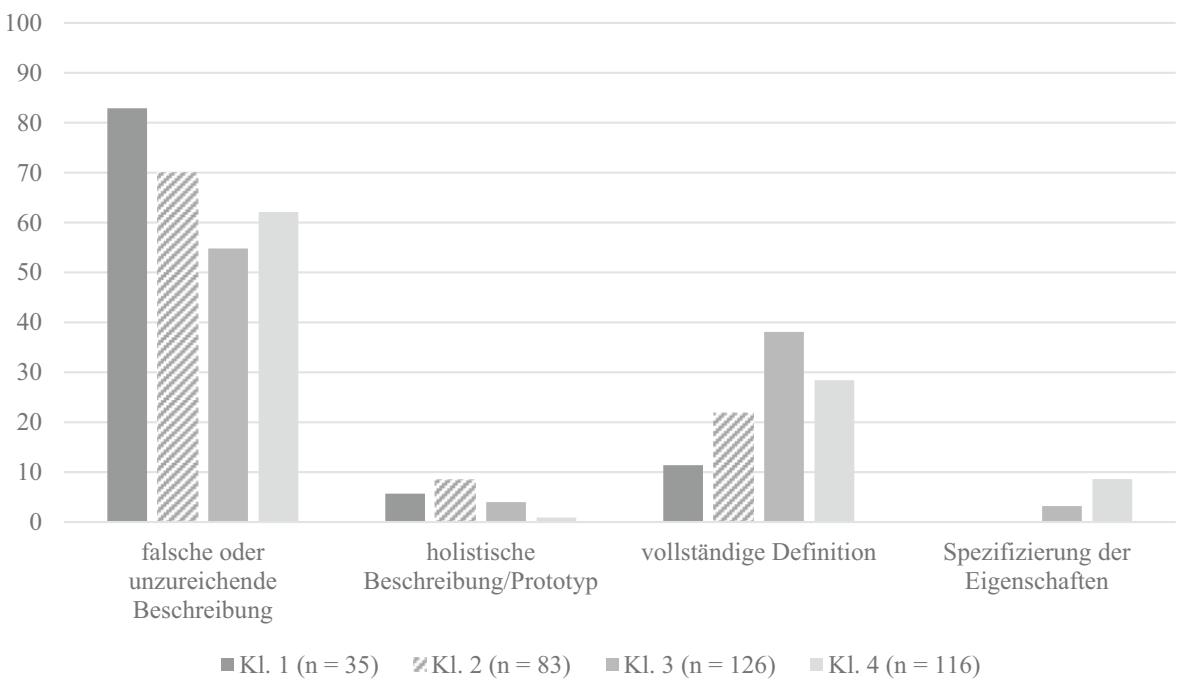

Abb. 9 Erklärungen des Begriffs Quadrat nach Klassenstufe getrennt, Angaben in \%

sentant identifiziert werden. Für die Figur ,unregelmäßiges Viereck' ergeben sich darüber hinaus signifikante Unterschiede zwischen den Klassenstufen 3 und 4, hier schneidet die Klassenstufe 4 besser ab. Die Lösungshäufigkeit der Figur ,Drachenviereck' unterscheidet sich zwischen den Klassenstufen 2 und 4 signifikant mit höheren korrekten Lösungsraten in Klassenstufe 4.

Zusammenfassend zeigt sich für den Begriffsumfang des Begriffs Quadrat, dass typische Nicht-Repräsentanten bereits ab Beginn der Grundschulzeit mehrheitlich sicher korrekt identifiziert werden. Haben die Nicht-Repräsentanten jedoch vier rechte Winkel, zeigen sich in den Klassenstufen 3/4 niedrigere Lösungsquoten als in den Klassenstufen 1/2. Für die übrigen Figuren ergibt sich ein unklares Bild: Tendenziell zeigen sich für die untypischen Nicht-Repräsentanten die niedrigsten Lösungsquoten in Klassenstufe 3; gleichzeitig zeigen sich in Klassenstufe 3 auch tendenziell die höchsten Lösungsquoten für die (un)typischen Repräsentanten.

Für alle Items des Begriffsinhalts des Begriffs Quadrat zeigen sich signifikante Unterschiede in den Lösungshäufigkeiten zwischen den Klassenstufen. Hinsichtlich des Items zur Erklärung des Begriffs Quadrats zeigt sich, dass in allen Klassenstufen über die Hälfte der Schülerinnen und Schüler eine unzureichende Erklärung des Begriffs notiert (s. Abb. 9). Die Varianzanalyse deckt einen signifikanten, aber schwachen Effekt der Klassenstufe $\left(F(3,356)=5,308, p=0,001, \eta^{2}=0,04\right)$ auf. Post-Hoc-Tests zeigen einen signifikanten Unterschied zwischen der Klassenstufe $1(M=0,28, S D=0,67)$ und den Klassenstufen $3(M=0,90, S D=1,02)$ und 4 $(M=0,84, S D=1,11)$ sowie zwischen den Lösungshäufigkeiten der Klassenstufen 2 $(M=0,52, S D=0,83)$ und 3 .

Bezüglich der Lösungshäufigkeit des Items zur begründeten Identifikation der Figur ,kleines Quadrat‘ als untypischer Repräsentant des Begriffs Quadrat zeigt sich ebenfalls ein signifikanter Unterschied zwischen den Klassenstufen $(F(3,413)=12,311, p<0,001)$ mit einem mittleren Effekt $\left(\eta^{2}=0,08\right)$. Post-Hoc- 
Tests zeigen, dass sich Klassenstufe $1(n=74, M=0,07, S D=0,25)$ von den übrigen Klassenstufen signifikant unterscheidet und Klassenstufe $2(n=96, M=0,28$, $S D=0,45)$ signifikant von Klassenstufe $3(n=128, M=0,45, S D=0,50)$, nicht jedoch von Klassenstufe $4(n=119, M=0,40, S D=0,49)$.

Hinsichtlich der Lösungshäufigkeiten der Items zu den Seiten- und Winkeleigenschaften des Quadrats zeigt sich ein signifikanter Unterschied zwischen den Klassenstufen mit mittlerem bzw. großem Effekt (Seite: $F(3,408)=16,218, p<0,001$, $\eta^{2}=0,11$; Winkel: $\left.F(3,406)=23,874, p<0,001, \eta^{2}=0,15\right)$. Für beide Items zeigen Post-Hoc-Tests, dass sich die Klassenstufen 3 (Seite: $n=107, M=0,76, S D=0,43$; Winkel: $n=107, M=0,73, S D=0,45$ ) und 4 (Seite: $n=121, M=0,79, S D=0,41$; Winkel: $n=121, M=0,73, S D=0,45)$ zu einer Gruppe zusammenfassen lassen. Bezüglich der Seiteneigenschaften lassen sich auch die Klassenstufen $1 \quad(n=82$, $M=0,44, S D=0,50)$ und $2(n=102, M=0,47, S D=0,50)$ zu einer Gruppe zusammenfassen. Beide Gruppen 1/2 und 3/4 unterscheiden sich hinsichtlich der Lösungshäufigkeit des Items zu den Seiteneigenschaften signifikant voneinander. Bezüglich der Winkeleigenschaften unterscheidet sich die Gruppe 3/4 signifikant von der Klassenstufe $1(n=81, M=0,24, S D=0,43)$ sowie von $2(n=101, M=0,53, S D=0,50)$. Zusätzlich unterscheiden sich auch die Klassenstufen 1 und 2 signifikant voneinander.

\subsection{Begriffsnetz}

Das Verständnis des Verhältnisses von Viereck - Rechteck bzw. Rechteck - Quadrat wurde explizit in zwei Items untersucht. Deskriptive Analysen zeigen, dass in den Klassestufen 1 ( $n=67$ Bearbeitungen) und 2 ( $n=70$ Bearbeitungen) keines der beiden Items richtig gelöst wurde. Die Inklusion der Begriffe Rechteck - Quadrat wird auch in den Klassenstufen 3 und 4 nur in 1,6\% $(n=130, S D=0,13)$ bzw. $5,0 \%(n=120, S D=0,15)$ der Bearbeitungen richtig erkannt; die Inklusion der Begriffe Viereck - Rechteck dagegen in $17 \%(n=130, S D=0,38)$ bzw. 36\% $(n=120$, $S D=0,48$ ). Insgesamt lösen sieben Lernende (sechs davon aus Klassenstufe 4 ) beide Items korrekt.

Entsprechend zeigt die Varianzanalyse einen signifikanten Unterschied zwischen den Klassenstufen für das Item zur Klasseninklusion von Viereck - Rechteck mit großem Effekt $\left(F(3,383)=22,862, p<0,001, \eta^{2}=0,15\right)$, nicht aber von Rechteck Quadrat $(F(3,361)=2,463, p=0,062)$. Post-Hoc-Tests zeigen für das Item zur Inklusion Viereck - Rechteck, dass die Klassenstufen 1 und 2 hinsichtlich ihres Verständnisses zusammengefasst werden können und sich signifikant von den Klassenstufen 3 und 4 unterscheiden. Zudem zeigt sich ein signifikanter Unterschied zwischen den Klassenstufen 3 und 4.

\section{Diskussion}

Ziel der Studie war eine detaillierte Deskription des Verständnisses der Begriffe Viereck, Rechteck und Quadrat in der Primarstufe und eine Suche nach Unterschieden zwischen einzelnen Klassenstufen, um weitere Annahmen zu Begriffsbildungspro- 
zessen hinsichtlich dieser Begriffe zu treffen. Im Folgenden werden zunächst die Limitationen der Studie aufgezeigt, um unter Berücksichtigung dieser die Ergebnisse zu diskutieren.

\subsection{Limitationen}

Zunächst soll betont werden, dass die Ergebnisse Einblicke in die Unterschiede zwischen Klassenstufen erlauben, das Design jedoch keine Schlussfolgerungen über Entwicklungsverläufe zulässt. Außerdem handelt es sich um eine Gelegenheitsstichprobe, die nicht zufällig zusammengesetzt, sondern in Klassen geklumpt ist. So konnte durch einen moderaten Ressourceneinsatz eine große Stichprobe erreicht werden. Gleichzeitig kann es dadurch zur Verzerrung der Ergebnisse kommen, da das Begriffsverständnis der Lernenden durch den Mathematikunterricht, der im Klassenverband erfolgt, geprägt ist. Um dieser Schwierigkeit zu begegnen, wurden 24 Klassen aus drei verschiedenen Schulen und in unterschiedlichen Bundesländern untersucht. Eine gewisse Verzerrung aufgrund der Unterrichtsvariablen (bspw. Lehrkraft, Schulbuch) kann dennoch nicht vollständig ausgeschlossen werden. Darüber hinaus könnten die Antworten der Schülerinnen und Schüler auch durch andere individuelle Faktoren als das Begriffsverständnis (bspw. Intelligenz, sprachliche Ausdrucksfähigkeit) beeinflusst sein. Da diese explorative Studie das Ziel verfolgte, Einblicke in das Verständnis ausgewählter Begriffe von Grundschülerinnen und Grundschülern zu geben und keine Kausalzusammenhänge untersucht, wurde von einer Kontrolle dieser Variablen abgesehen. Aufgrund der geklumpten Stichprobe und der fehlenden Kontrolle dieser Variablen sind die Ergebnisse diese Studie zwar nicht verallgemeinerbar, geben aber dennoch detaillierte Einblicke in das Verständnis der ebenen Figurenbegriffe Viereck, Rechteck und Quadrat.

Um das Verständnis der Begriffe Viereck, Rechteck und Quadrat in verschiedenen Klassenstufen zu erfassen, wurden standardisierte Items entwickelt. Dieser quantitative Zugang hat den Vorteil, dass Unterschiede zwischen den Klassenstufen auf einer allgemeinen Ebene sichtbar werden. Da die drei Indikatoren Begriffsumfang, -inhalt und -netz aus theoretischer Perspektive voneinander abhängen und verschiedene Items mit unterschiedlichen individuellen Begriffskonzepten richtig gelöst werden können, wurden in dieser Studie zunächst Einzelitems und keine Skalen betrachtet. Dabei bleiben mögliche Zusammenhänge zwischen den Items allerdings unberücksichtigt.

Die entwickelten Testitems erwiesen sich in Vorstudien größtenteils als geeignet zur Erfassung des Verständnisses der drei Begriffe. Einzig die geschlossenen Items zur Erfassung des Eigenschaftswissen in Bezug auf die Seiten zeigen auch in der Pilotierung noch Schwierigkeiten und wurden für die Hauptstudie erneut überarbeitet. Deskriptive Analysen mit den Daten der Hauptstudie ergeben, dass für alle drei Figuren die Antwortmöglichkeit ,Alle Seiten sind gleich lang ' am häufigsten ausgewählt wurde (Ausnahme: Rechteck Klassenstufe 1, hier tritt die Antwortoption ,weiß ich nicht' ebenso häufig auf). Gleichzeitig stimmen der Antwortoption ,Je zwei Seiten sind gleich lang' prozentual die meisten Lernenden bei der Figur Rechteck zu und der Antwortoption ,Das ist egal' bei der Figur Viereck. Dies deutet daraufhin, dass diese Antworten von einem Teil der Lernenden als richtige Option erkannt werden. 
Dass ca. $20 \%$ der Lernenden (überwiegend aus den Klassenstufen 1/2) für die Figuren Rechteck und Quadrat die Antwortoption ,weiß ich nicht' wählen, deutet darauf hin, dass diese Antwortoption genutzt wird, statt zu raten. Unklar bleibt jedoch, ob die geringe Lösungsquote des Items für den Begriff Rechteck (ca. 20\%), auf fehlendes Wissen oder die komplexe Formulierung der richtigen Antwort zurückzuführen ist. Mittelwertunterschiede zugunsten der Kinder, die angeben, eine weitere Sprache als Deutsch zu sprechen, sprechen eher dafür, dass das Item das Wissen der Kinder und nicht sprachliche Fähigkeiten misst. Zusammenfassend wird daher zunächst davon ausgegangen, dass sich die Items mit Blick auf das Wissen der Lernenden zu den Seiteneigenschaften valide interpretieren lassen.

Zusammengenommen zeigt die kritische Auseinandersetzung mit dem methodischen Vorgehen in dieser Studie einerseits Grenzen im Hinblick auf die Verallgemeinerbarkeit der Ergebnisse und andererseits weiteren Forschungsbedarf mit Blick auf die eingesetzten Instrumente auf.

\subsection{Diskussion der Ergebnisse}

Trotz der angeführten Limitationen geben die dargestellten Ergebnisse detaillierte und bisher für den deutschsprachigen Raum nicht berichtete Einblicke in das Verständnis der Begriffe Viereck, Rechteck und Quadrat in der Primarstufe, die erste Hypothesen über deren individuelle Begriffskonzepte zulässt und dadurch spezifische Auffälligkeiten in Begriffsbildungsprozessen offenbart.

\subsubsection{Diskussion der Ergebnisse zum Begriff Viereck}

Für den Begriff Viereck zeigt sich sowohl in den Lösungsquoten der Items zum Begriffsumfang als auch zum Begriffsinhalt ein qualitativer Sprung zwischen den Klassenstufen 2 und 3. Bezüglich des Begriffsinhalts ist bemerkenswert, dass eher jüngere Kinder Definitionen des Begriffs angeben, die dem formal-logischen Begriffskonzept nahe kommen, während in allen anderen Items zum Begriffsinhalt die Lernenden der Klassenstufen 3 besser abschneiden als die der Klassenstufe 2. Die Ergebnisse weisen darauf hin, dass die Lernenden der Klassenstufen 3/4 zwar stärker die definierenden Eigenschaften des Begriffs Viereck fokussieren, die individuellen Begriffskonzepte aber gleichzeitig prototypisch (Medin und Smith 1984; Rosch 1978) geprägt sein könnten.

Weitere Anhaltspunkte für ein prototypisch geprägtes Verständnis des Begriffs Viereck zeigen sich in den Ergebnissen zum Begriffsumfang: Typische Repräsentanten (in diesem Fall Quadrate) werden häufiger korrekt identifiziert als untypische Repräsentanten. Dabei zeigen sich bezüglich der untypischen Repräsentanten Unterschiede zugunsten der höheren Klassenstufen. Im Einklang mit den Ergebnissen vorheriger Studien (Grassmann et al. 2002; Höglinger und Senftleben 1997; Reemer und Eichler 2005; Unterhauser 2020) zeigt sich auch in dieser Studie, dass das Rechteck mindestens in den Klassenstufen 1 und 2 kein prototypisches Viereck ist.

In der Verknüpfung der Ergebnisse zum Begriffsinhalt und Begriffsumfang finden sich Hinweise auf ein gleichzeitiges eigenschaftsbezogenes und ganzheitliches Verständnis (s. Abschn. 4): So wird das ,konkave Viereck` als untypischer Repräsentant 
des Begriffs Viereck in allen Klassenstufen im reinen Identifikations-Item deutlich seltener als Repräsentant ausgewählt als in dem entsprechenden Begründungs-Item. Daraus kann geschlossen werden, dass die Schülerinnen und Schüler - bei geeigneter Aufgabenstellung (Battista 2007; Clements et al. 1999; Fuys et al. 1988) diesen untypischen Repräsentanten als Viereck akzeptieren und ihre Identifikation auch anhand von Eigenschaften richtig begründen, obwohl sie die Figur zunächst nicht als Repräsentant akzeptieren. Daraus kann vorsichtig gefolgert werden, dass Lernende nicht entweder ein eigenschaftsbezogenes oder ein ganzheitliches Verständnis des Begriffs Viereck ausgebildet haben, sondern beide Verständnisweisen parallel auftreten.

\subsubsection{Diskussion der Ergebnisse zum Begriff Rechteck}

Für den Begriff Rechteck zeigen sich vergleichsweise geringe Unterschiede zwischen den Klassenstufen. Hinsichtlich des Begriffsinhalts zeigt sich ähnlich zu vorhergehenden Studien (Aktaş Arnas und Aslan 2010; Clements et al. 1999; Reemer und Eichler 2005) eine starke prototypische Prägung der Erklärungen in allen Klassenstufen. Auffällig ist in allen Items zum Begriffsinhalt, dass die Lernenden den Winkeleigenschaften wenig Bedeutung beimessen und, wie auch in vorangegangenen Studien gezeigt, vor allem die Seitenverhältnisse betrachten (Aktaş Arnas und Aslan 2010; Burger und Shaughnessy 1986; Clements et al. 1999; Maier 2019; Žilková und Kopáčová 2018).

Hinsichtlich des Begriffsumfangs gilt, dass Nicht-Repräsentanten, die größtenteils die Eigenschaft der Parallelität der Seitenpaare verletzen, sowie typische Repräsentanten in allen Klassenstufen sehr häufig korrekt identifiziert werden; Quadrate als untypische Repräsentanten dagegen nur sehr selten. Im Widerspruch zu bisherigen empirischen Ergebnissen (Aktaş Arnas und Aslan 2010; Aslan und Aktaş Arnas 2007; Clements et al. 1999; Maier 2019; Unterhauser 2020) zeigt sich sowohl ein Einfluss der Orientierung parallel zum Blattrand als auch der konkreten Form der Figur auf die Lösungsrate, da sich in den Klassenstufen 1, 2 und 4 bei der Figur ,Rechteck auf der Spitze' und für die Klassenstufe 1-4 bei der Figur ,schmales Rechteck' niedrigere Lösungsraten zeigen als bei der Figur ,prototypisches Rechteck‘. Unterschiede zwischen den Klassenstufen ergeben sich nur bei fünf Figuren zwei davon sind untypische Repräsentanten, zwei untypische Nicht-Repräsentanten. Im Vergleich fällt auf, dass die Figur, offene Seite' häufig fälschlicherweise als Repräsentant des Begriffs Rechteck ausgewählt wird, obwohl diese Figur selten als Repräsentant des Begriffs Viereck gewählt wird. Dies könnte die These stützen, dass die Schülerinnen und Schüler die Figur - zumindest bis in die dritte Klasse - eher ganzheitlich betrachten und daher als Rechteck identifizieren.

Insgesamt deuten die Daten im Einklang mit der prototypischen Theorie (Medin und Smith 1984; Rosch 1978) darauf hin, dass individuelle Begriffskonzepte der Lernenden zum Begriff Rechteck in allen Klassenstufen eher prototypisch geprägt sind. Hinweise für eine Weiterentwicklung dieser individuellen Begriffskonzepte im Sinne der Stufenmodelle (van Hiele 1986; Vollrath 1984; Winter 1983) zeigen die Daten nur an vereinzelten Stellen. Bspw. wird das Item zu den Seiteneigenschaften in der dritten Klassenstufe (nicht jedoch in Klassenstufe 4) häufiger korrekt 
gelöst und untypische Repräsentanten sowie einzelne untypische Nicht-Repräsentanten in höheren Klassenstufen häufiger korrekt identifiziert. Die Tatsache, dass für den Begriff Rechteck definierende Eigenschaftsbegriffe (bspw. rechtwinklig) erst in den Klassenstufen 3 bzw. 4 thematisiert werden (Niedersächsisches Kultusministerium 2017), der Begriff Rechteck nach eigenen Analysen in vielen Schulbüchern jedoch bereits ab Klassenstufe 1 - meist in prototypischer Darstellung - verwendet wird, könnte hinderlich für die Entwicklung eines umfassenden Verständnisses des Begriffs Rechteck sein.

\subsubsection{Diskussion der Ergebnisse zum Begriff Quadrat}

In der Aufgabe zum Begriffsumfang zeigen sich vergleichsweise niedrige Lösungsquoten für die Repräsentanten des Begriffs Quadrat. Dies widerspricht bisherigen Ergebnissen, die für den Begriff Quadrat in der Regel höhere Lösungsraten zeigen als für den Begriff Rechteck (Aktaş Arnas und Aslan 2010; Aslan und Aktaş Arnas 2007; Clements et al. 1999; Kyriakides 1999; Reemer und Eichler 2005). Dabei fällt auf, dass die Orientierung parallel zum Blattrand der Figur ebenfalls entgegen bisheriger empirischer Ergebnisse (Aktaş Arnas und Aslan 2010; Aslan und Aktaş Arnas 2007; Burger und Shaughnessy 1986; Clements et al. 1999; Fuys et al. 1988; Hannibal 1999; Yin 2003) keinen Einfluss auf die Lösungsrate zu haben scheint, da die Lösungsraten für die Figur, Quadrat auf der Spitze“ jeweils nur knapp unter denen der Figur ,prototypisches Quadrat' liegen; die Größe der Figur dagegen schon (Lösungsraten für Figur ,kleines Quadrat' liegen deutlich unter denen der Figur ,prototypisches Quadrat'). Insgesamt unterscheidet sich die Klassenstufe 4 in den Lösungsraten der Items zum Begriffsumfang am grundsätzlichsten von den übrigen Klassenstufen, jedoch ergibt sich kein einheitliches Bild: Beispielsweise wählen Lernende der 4. Klassenstufe einzelne untypische Nicht-Repräsentanten seltener (bspw. ,abgerundete Ecken'), aber häufiger fälschlicherweise Rechtecke als Repräsentanten des Begriffs Quadrat aus. Die Ursache dafür könnte in einem beginnenden, aber noch nicht gefestigten Verständnis der Klasseninklusion liegen, welches dazu führen könnte, dass auch Rechtecke fälschlicherweise als Quadrate betrachtet werden.

Für das Verständnis des Begriffsinhalts zeigen sich bedeutsame Unterschiede zwischen den Klassenstufen, insbesondere zwischen den Klassenstufen 2 und 3/4, wobei die Schülerinnen und Schüler in den Klassenstufen 3/4 höhere Lösungsraten zeigen. Gleichzeitig geben jedoch viele Lernende der Klassenstufen 3/4 unvollständige Definitionen an, die nur die Seiteneigenschaften des Begriffs berücksichtigen.

Zusammenfassend kann vermutet werden, dass - ähnlich zu dem Begriff Rechteck - bei vielen Schülerinnen und Schülern insbesondere in den Klassenstufen 3/4 sowohl ein eigenschaftsbezogenes als auch ein prototypisches Verständnis des Begriffs Quadrat vorherrscht. Die vorliegenden Daten lassen dabei teilweise vermuten, dass die individuellen Begriffskonzepte zu dem Begriff Quadrat über lange Zeit stark prototypisch geprägt sind (Fujita 2012). Gleichzeitig ergeben sich Hinweise darauf, dass sich prototypisches und eigenschaftsbezogenes Verständnis zeitlich versetzt entwickeln könnte. Dies zeigt sich bspw. in den Ergebnissen des Identifikations- und Begründungsitems zur Figur ,kleines Quadrat': Während Schülerinnen und Schüler der Klassenstufe 1 und 2 das Item zur begründeten Identifikation deutlich seltener 
korrekt lösen, also seltener korrekte Identifikationen mit korrekten Begründungen stützen, nähern sich die Lösungsraten der beiden Items in den Klassenstufen 3 und 4 an.

\subsubsection{Diskussion der Ergebnisse zum Begriffsnetz}

Mit Blick auf die Klasseninklusion zeigt sich ein heterogenes Bild: Während die Inklusion der Begriffe Viereck - Rechteck mehr als einem Drittel der Schülerinnen und Schüler der Klassenstufe 4 gelingt, scheint die Inklusion der Begriffe Rechteck - Quadrat für Lernende aller Klassenstufen eine große Herausforderung zu sein. Dieses Bild deckt sich mit den Ergebnissen vorheriger Studien ebenso wie mit den Erkenntnissen dieser Studie, die in den Aufgaben zum Begriffsumfang gewonnen wurden. Erkenntnisse zum Begriffsumfang zeigen, dass Kinder den Begriff Viereck partitional klassifizieren (Grassmann et al. 2002; Höglinger und Senftleben 1997, Unterhauser 2020) und vor allem die Begriffe Quadrat und Rechteck als voneinander getrennt ansehen (Burger und Shaughnessy 1986; Clements et al. 1999; Fuys et al. 1988; Heinze 2002a, b; Höglinger und Senftleben 1997; Koleza und Giannisi 2013; Maier 2019; Reemer und Eichler 2005; Vinner und Hershkowitz 1980; Yin 2003). Zusätzlich lassen die gefundenen individuellen Begriffskonzepte der Lernenden zu den einzelnen Begriffen Viereck, Rechteck und Quadrat auch gewisse Hürden für den Aufbau eines klasseninklusiven Begriffsverständnisses erkennen: Lernende nehmen zwar im Verlauf der Grundschulzeit Figuren stärker eigenschaftsbezogen wahr (bspw. die Seiteneigenschaften des Rechtecks), legen dabei die prototypische Prägung aber nicht vollständig ab (bspw. Figur ,offene Seite ' wird weiter als Rechteck identifiziert). Dies kann dazu führen, dass Lernende Figuren zwar nicht mehr (ausschließlich) ganzheitlich betrachten, jedoch nicht-definierende Eigenschaften (bspw. ein Rechteck hat zwei lange und zwei kurze Seiten) in ihre individuellen Begriffskonzepte integrieren, wie es auch Fujita und Jones (2007) für ältere Schülerinnen und Schüler beschreiben. Diese Vorstellung ist hinderlich für ein Verständnis der Klasseninklusion Quadrat - Rechteck.

\subsubsection{Schlussfolgerungen aus den Ergebnissen}

Die Ergebnisse der vorliegenden Studie geben Hinweise darauf, dass sich die individuellen Begriffskonzepte von Lernenden zu den Begriffen Viereck, Rechteck und Quadrat bereits im Laufe der Grundschulzeit als eigenschaftsbezogen, aber prototypisch geprägt erweisen. Darin könnten Schwierigkeiten im Begriffsverständnis in der Sekundarstufe begründet sein, wie bereits in vorherigen Studien vermutet wurde (Fujita 2012; Gunčaga und Žilková 2019) und sich auch in Studien zu geometrischen Körpern zeigt (Wöller 2020).

Diese Ergebnisse könnten die These stützten, dass Kinder gleichzeitig über unterschiedliche Arten, Begriffe zu verstehen, verfügen, die miteinander konkurrieren und im Sinne überlappender Wellen interagieren (Chen und Siegler 2000, s. Abschn. 4). Alternativ könnten die Ergebnisse einen U-förmigen Entwicklungsverlauf nahelegen (Strauss 1982; Siegler 2004). Dabei wird u. a. davon ausgegangen, dass Lernende bei der Entwicklung neuer, systematischer Ansätze zur Lösung von Problemen 
diesem Ansatz Vorrang einräumen und dadurch Rückschritte in ihrer Leistung in Bezug auf spezifische Probleme zeigen (bspw. Siegler 2004). Übertragen auf das Begriffsverständnis der Begriffe Viereck, Rechteck und Quadrat könnte das stärker eigenschaftsbezogene Verständnis der Figuren im Laufe der Grundschulzeit dazu führen, nicht nur definierende, sondern auch charakteristische Eigenschaft als notwendig für einen Begriff zu betrachten. Dies würde wiederrum zu weniger korrekten Identifikationen von insbesondere untypischen (Nicht-)Repräsentanten bzw. stärker prototypisch geprägten Erklärung des Begriffs führen. Anders als bei dem Modell der überlappenden Wellen würde das Modell der U-förmigen Entwicklung dabei von einer Weiterentwicklung des Begriffsverständnisses ausgehen und nicht von einem Fortbestehen vorheriger Lösungsansätze. Weitere Forschung ist notwendig, um $\mathrm{zu}$ untersuchen, wann und warum sich prototypisch geprägte individuelle Begriffskonzepte ausbilden und wie der Übergang zu einer stärker eigenschaftsbezogenen Wahrnehmung der ebenen Figuren im Laufe der Grundschulzeit genutzt werden kann, um die prototypische Sichtweise abzulegen bzw. ihr vorzubeugen.

Darüber hinaus stellt sich aus didaktischer Perspektive die Frage, welche Bedingungen bereits im Elementarbereich bzw. im Geometrieunterricht der Grundschule dazu führen, dass Lernende ein prototypisches Verständnis der Begriffe entwickeln. Als mögliche Hürde wurde die versetzte Einführung der Begriffe Viereck, Rechteck und Quadrat und der Eigenschaftsbegriffe rechtwinklig und parallel ausgemacht, die Lehrkräfte dazu verleiten kann, diese Eigenschaften der Figuren in der Klassenstufe $1 / 2$ nicht zu thematisieren - auch wenn dies alltagssprachlich möglich wäre (Bartolini Bussi und Baccaglini-Frank 2015). In vielen Materialien der Grundschule und des Elementarbereichs sind zudem vorwiegend typische Repräsentanten der Begriffe Rechteck und Quadrat zu finden, ebenso wie eine partitionale Klassifizierung der beiden Begriffe. Auf Grundlage unserer Studie scheint es wichtig, von Anfang an und im Besonderen im Geometrieunterricht darauf zu achten, dass Lernende einerseits mit typischen und untypischen Repräsentanten und Nicht-Repräsentanten konfrontiert werden und andererseits selbst verschiedene Figuren erzeugen, manipulieren und miteinander vergleichen, damit anschlussfähige Begriffsbildungsprozesse ermöglicht werden. 


\section{Anhang}

Tab. 5 Codierung des Items ,Erklärung des Begriffs Viereck/Rechteck/Quadrat“ (Begriffsinhalt)

\begin{tabular}{|c|c|c|c|c|c|}
\hline Code & $\begin{array}{l}\text { Codebe- } \\
\text { zeichnung }\end{array}$ & Codebeschreibung & $\begin{array}{l}\text { Ankerbeispiele } \\
\text { Viereck }\end{array}$ & $\begin{array}{l}\text { Ankerbeispiele } \\
\text { Rechteck }\end{array}$ & Ankerbeispiele Quadrat \\
\hline 0 & $\begin{array}{l}\text { Falsche } \\
\text { oder } \\
\text { unzu- } \\
\text { rei- } \\
\text { chende } \\
\text { Erklä- } \\
\text { rung }\end{array}$ & $\begin{array}{l}\text { Die Erklärung ist falsch } \\
\text { oder enthält nicht alle } \\
\text { zur eindeutigen Be- } \\
\text { stimmung des Begriffs } \\
\text { notwendigen Eigen- } \\
\text { schaften }\end{array}$ & $\begin{array}{l}\text { „Ein Viereck ist } \\
\text { eine Form, die } \\
\text { Ecken hat, aber } \\
\text { es ist egal, ob es } \\
\text { schief steht oder } \\
\text { ob es gerade } \\
\text { steht.“ } \\
\text { „Ein Viereck hat } \\
\text { acht Ecken und } \\
\text { sechs Platten.“ }\end{array}$ & $\begin{array}{l}\text { „Ein Rechteck hat } \\
\text { gerade Linien und } \\
\text { vier Ecken.“ } \\
\text { „Es ist lang und } \\
\text { groß!“ }\end{array}$ & $\begin{array}{l}\text { „Ein Quadrat ist fast } \\
\text { wie ein Viereck nur } \\
\text { länger.“ } \\
\text { „Ein Quadrat ist ein } \\
\text { bisschen kleiner als das } \\
\text { Rechteck.“ }\end{array}$ \\
\hline 1 & $\begin{array}{l}\text { Alltags- } \\
\text { beispiel, } \\
\text { ganz- } \\
\text { heit- } \\
\text { liche } \\
\text { Erklä- } \\
\text { rung, } \\
\text { Proto- } \\
\text { typ }\end{array}$ & $\begin{array}{l}\text { Die Erklärung nimmt } \\
\text { Bezug auf ein Alltags- } \\
\text { beispiel, den ganzheit- } \\
\text { lichen Eindruck der } \\
\text { Figur oder umfasst } \\
\text { neben definierenden } \\
\text { Eigenschaften auch } \\
\text { nicht-definierende Ei- } \\
\text { genschaften, die auf } \\
\text { ein prototypisches Ver- } \\
\text { ständnis des Begriffs } \\
\text { hinweisen }\end{array}$ & $\begin{array}{l}\text { „Ein Viereck } \\
\text { sieht so aus wie } \\
\text { ein Kissen.“ } \\
\text { „Ein Viereck } \\
\text { hat vier Ecken, } \\
\text { die Ecken sind } \\
\text { gleich groß.“ } \\
\text { „Ein Viereck hat } \\
\text { vier gleich lange } \\
\text { Seiten und vier } \\
\text { Ecken.“ }\end{array}$ & $\begin{array}{l}\text { „Ist wie eine } \\
\text { Schultasche oder } \\
\text { ein Block.“ } \\
\text { „Ein Rechteck } \\
\text { sieht fast aus wie } \\
\text { ein Viereck. nur } \\
\text { dass ein Rechteck } \\
\text { langgezogen ist.“ } \\
\text { „Ein Rechteck hat } \\
\text { zwei lange Seiten } \\
\text { und zwei kurze.“ }\end{array}$ & $\begin{array}{l}\text { „Ist so wie Fenster.“ } \\
\text { „Ein Quadrat sieht so } \\
\text { aus [Bild von Quadrat } \\
\text { gezeichnet].“ } \\
\text { „Ein Quadrat sieht wie } \\
\text { ein kleines Blatt.“ }\end{array}$ \\
\hline 2 & $\begin{array}{l}\text { Bezug } \\
\text { zu zen- } \\
\text { tralen } \\
\text { Eigen- } \\
\text { schaf- } \\
\text { ten des } \\
\text { Begriffs }\end{array}$ & $\begin{array}{l}\text { Die Erklärung nimmt } \\
\text { Bezug auf zentrale } \\
\text { definierende Eigen- } \\
\text { schaften des Begriffs. } \\
\text { für den Begriff Viereck } \\
\text { ist damit eine eindeu- } \\
\text { tige Bestimmung des } \\
\text { Begriffs möglich }\end{array}$ & $\begin{array}{l}\text { „Ein Viereck } \\
\text { hat vier Ecken } \\
\text { und gibt's in } \\
\text { verschiedenen } \\
\text { Größen und } \\
\text { Formen.“ } \\
\text { „Ein Viereck hat } \\
\text { vier Seiten.“ } \\
\text { „Ein Vireck hat } \\
\text { Virecken. Es ist } \\
\text { Nicht Rund.“ }\end{array}$ & $\begin{array}{l}\text { „Ein Rechteck hat } \\
\text { vier zwei Seiten } \\
\text { gleich lang, die } \\
\text { anderen Seiten } \\
\text { auch gleich lang.“ }\end{array}$ & $\begin{array}{l}\text { „Ein Quadrat ist ein } \\
\text { Viereck und hat vier } \\
\text { Ecken, alles Seiten sind } \\
\text { gleich lang.“ } \\
\text { „Ein Quadrat hat vier } \\
\text { gleich lange Linien.“ } \\
\text { „Ein Quadrat hat vier } \\
\text { gleiche Ecken.“ }\end{array}$ \\
\hline 3 & $\begin{array}{l}\text { Vollständige } \\
\text { Defini- } \\
\text { tion } \\
\text { bzw. } \\
\text { Spezifi- } \\
\text { zierung } \\
\text { der } \\
\text { Eigen- } \\
\text { schaf- } \\
\text { ten }\end{array}$ & $\begin{array}{l}\text { Die Erklärung nimmt } \\
\text { Bezug auf zentrale defi- } \\
\text { nierende Eigenschaften } \\
\text { des Begriffs, sodass } \\
\text { der Begriff eindeutig } \\
\text { bestimmt werden kann. } \\
\text { Für den Begriff Viereck } \\
\text { werden die definie- } \\
\text { renden Eigenschaften } \\
\text { weiter spezifiziert }\end{array}$ & $\begin{array}{l}\text { „Ein Viereck hat } \\
\text { vier Ecken. Die } \\
\text { Seiten können } \\
\text { gleich lang } \\
\text { sein, aber auch } \\
\text { unterschiedlich } \\
\text { lang. Seiten } \\
\text { könne gerade } \\
\text { aber auch schräg } \\
\text { sein.“ }\end{array}$ & $\begin{array}{l}\text { „Ein Rechteck } \\
\text { hat gegenüberlie- } \\
\text { gende Seiten. Die } \\
\text { Parallelen sind } \\
\text { immer gleichlang. } \\
\text { Es hat vier rechte } \\
\text { Winkel.“ }\end{array}$ & $\begin{array}{l}\text { „Ein Quadrat hat vier } \\
\text { gleiche Ecken und vier } \\
\text { gleiche Seiten.“ } \\
\text { „Ein Quadrat, das ist so } \\
\text { wie den Rechteck, aber } \\
\text { ein bisschen anders, die } \\
\text { hat die Seiten gleich } \\
\text { lang.“ } \\
\text { „Ein Quadrat hat auch } \\
\text { vier rechte Winkel } \\
\text { und auch gleich lange } \\
\text { Seiten.“ }\end{array}$ \\
\hline
\end{tabular}




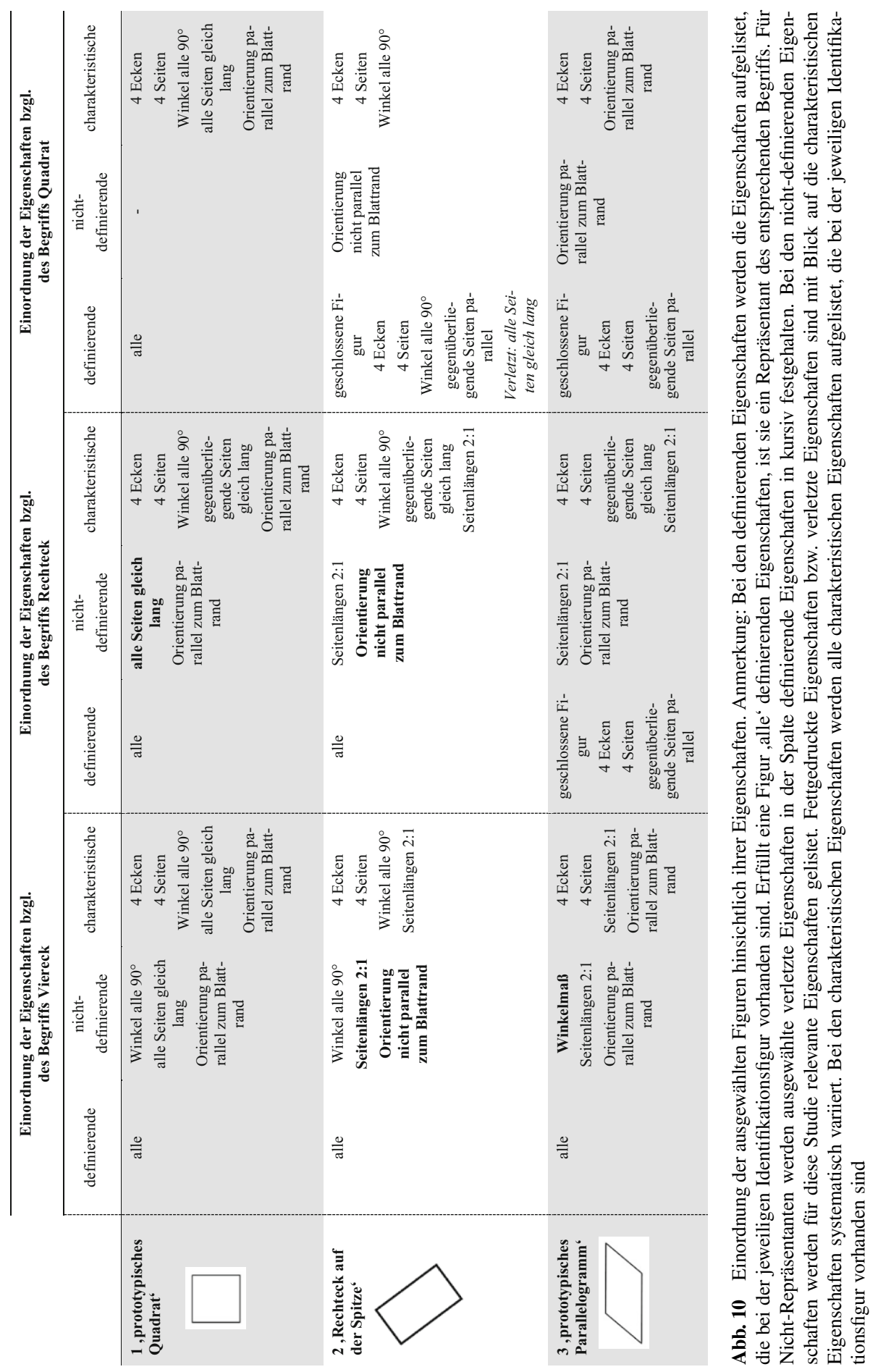




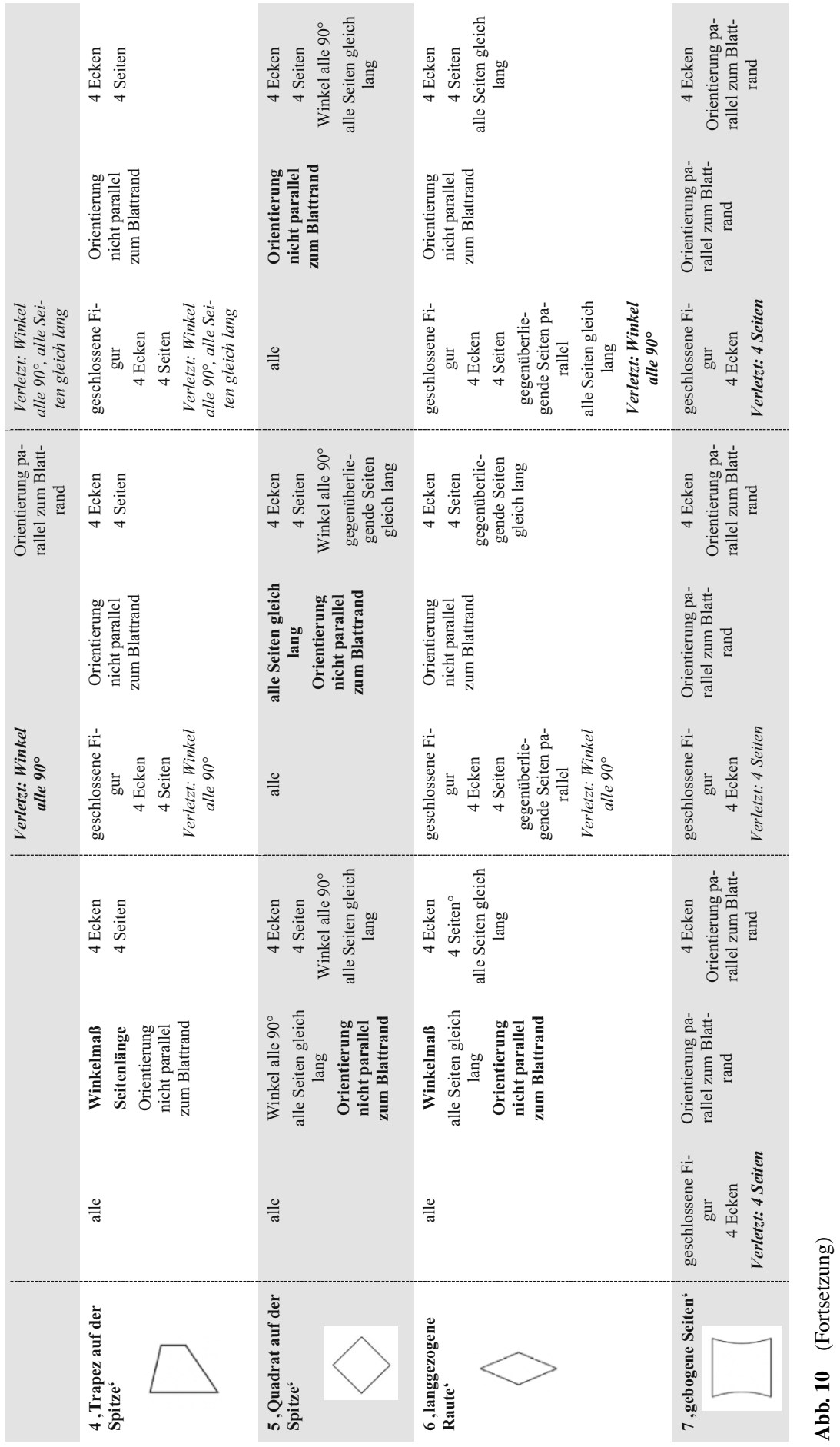




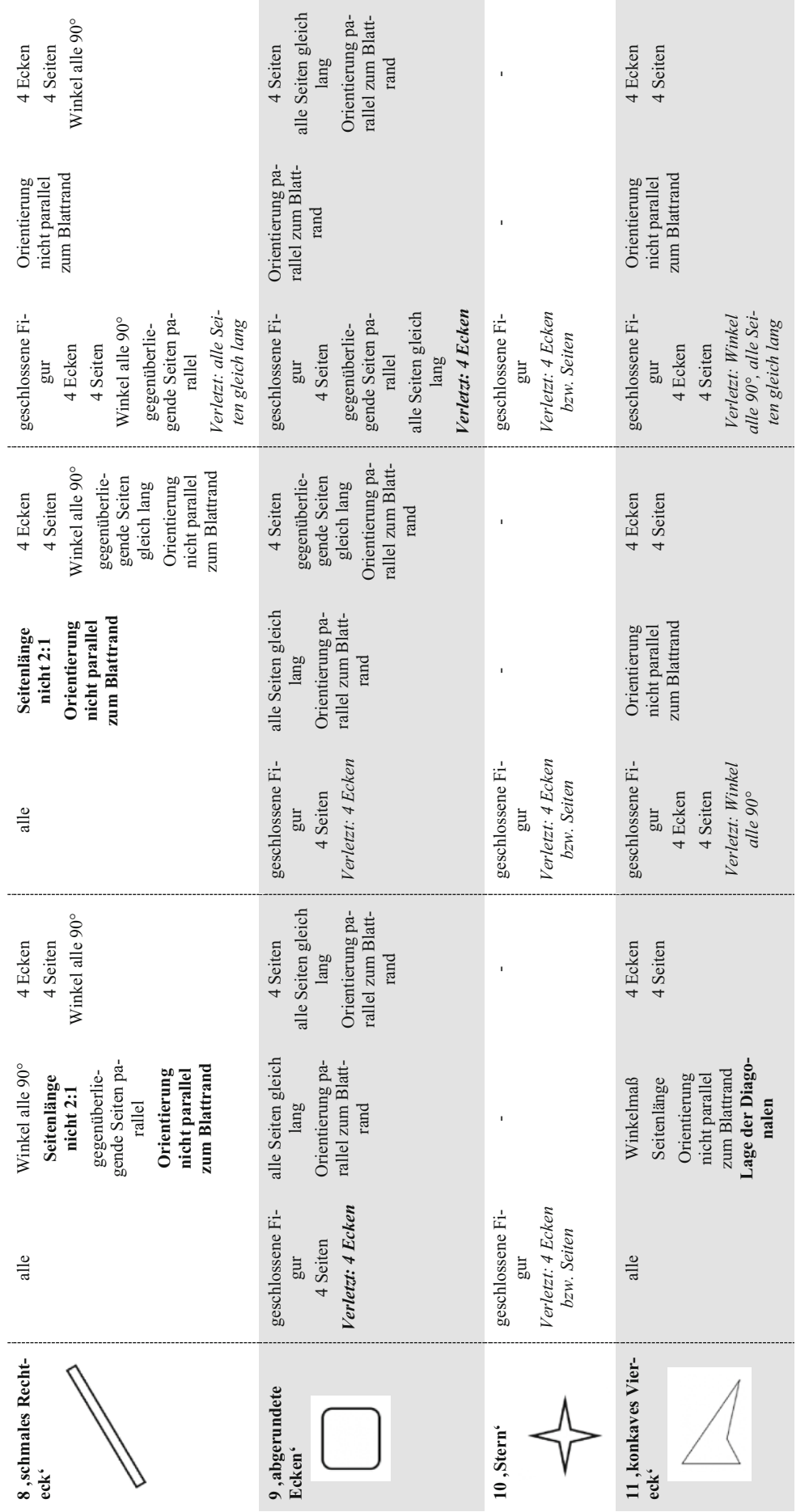

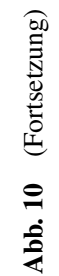




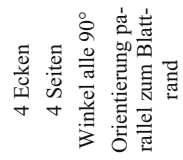

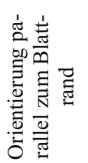
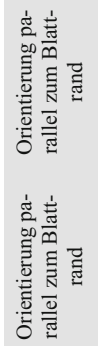

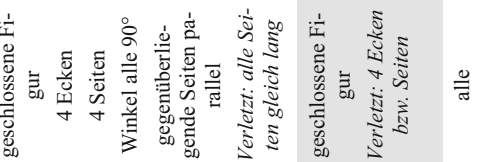

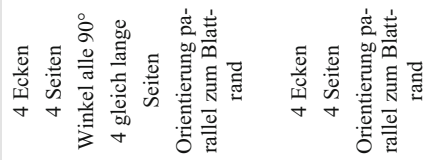
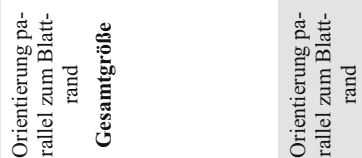

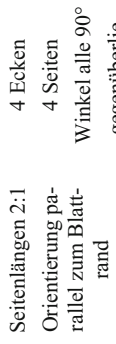

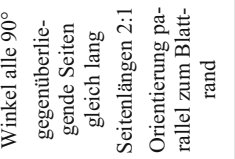

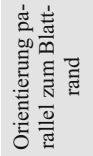

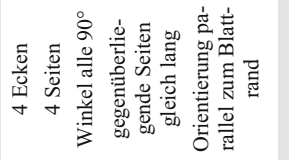

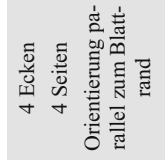

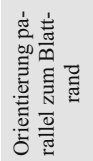

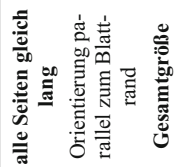

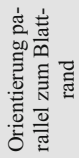

$\stackrel{\frac{0}{\bar{*}}}{2}$

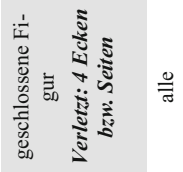

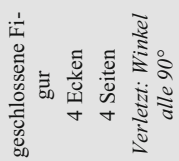

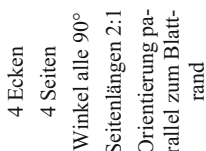

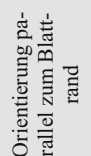

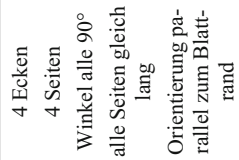

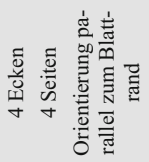

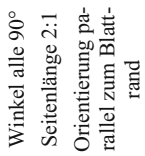

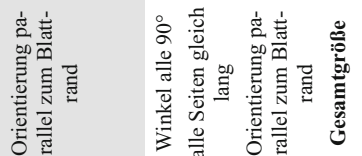
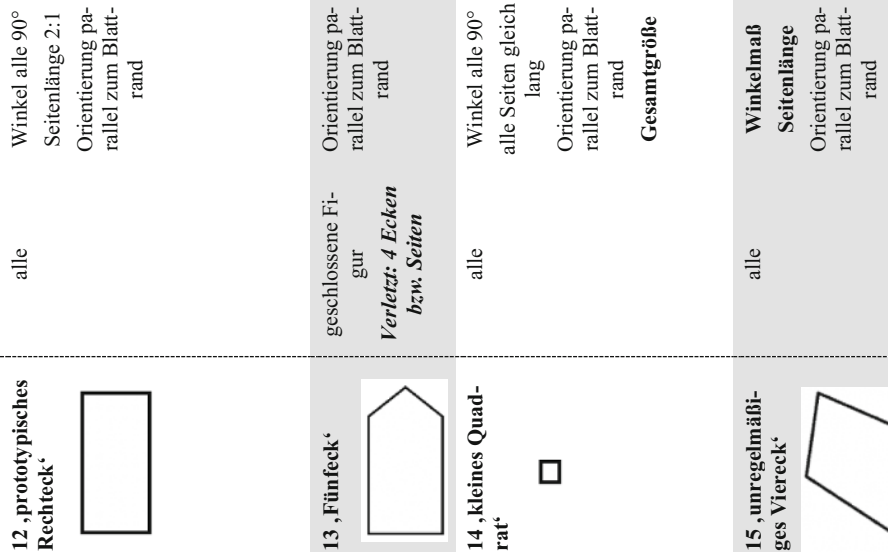

ڤ

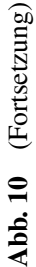




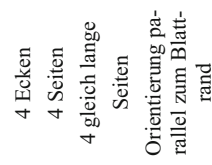

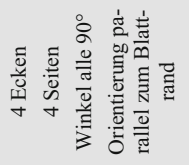

辈受

空

竞

总苇 范

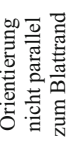

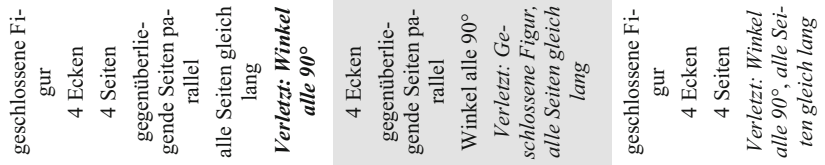

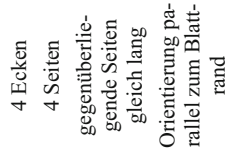

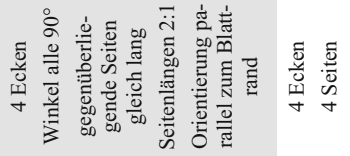

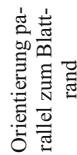

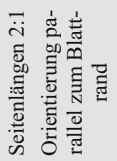

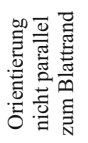

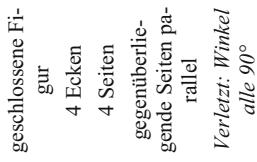

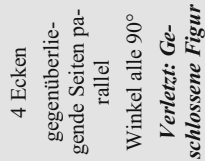

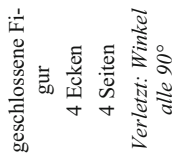

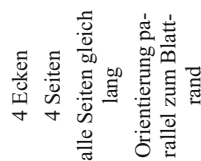

을

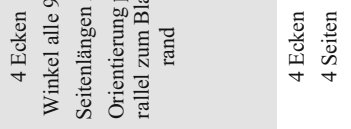

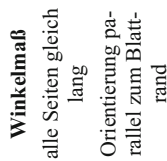

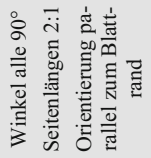

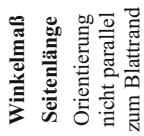
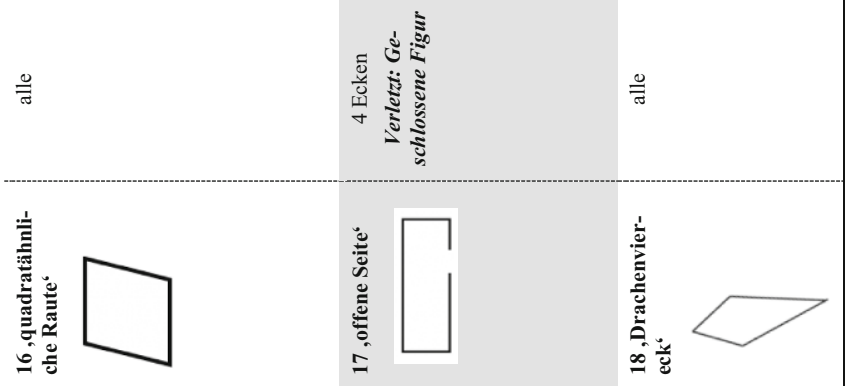

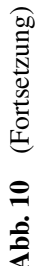


Funding Open Access funding enabled and organized by Projekt DEAL.

Open Access Dieser Artikel wird unter der Creative Commons Namensnennung 4.0 International Lizenz veröffentlicht, welche die Nutzung, Vervielfältigung, Bearbeitung, Verbreitung und Wiedergabe in jeglichem Medium und Format erlaubt, sofern Sie den/die ursprünglichen Autor(en) und die Quelle ordnungsgemäß nennen, einen Link zur Creative Commons Lizenz beifügen und angeben, ob Änderungen vorgenommen wurden.

Die in diesem Artikel enthaltenen Bilder und sonstiges Drittmaterial unterliegen ebenfalls der genannten Creative Commons Lizenz, sofern sich aus der Abbildungslegende nichts anderes ergibt. Sofern das betreffende Material nicht unter der genannten Creative Commons Lizenz steht und die betreffende Handlung nicht nach gesetzlichen Vorschriften erlaubt ist, ist für die oben aufgeführten Weiterverwendungen des Materials die Einwilligung des jeweiligen Rechteinhabers einzuholen.

Weitere Details zur Lizenz entnehmen Sie bitte der Lizenzinformation auf http://creativecommons.org/ licenses/by/4.0/deed.de.

\section{Literatur}

Aktaş Arnas, Y., \& Aslan, D. (2010). Children's classification of geometric shapes. Ç. ̈̈. Sosyal Bilimler Enstitüsü Dergisi, 19(1), 254-270.

Aslan, D., \& Arnas, A. Y. (2007). Three- to six-year-old children's recognition of geometric shapes. International Journal of Early Years Education, 15(1), 83-104.

Bartolini Bussi, M. G., \& Baccaglini-Frank, A. (2015). Geometry in early years. sowing seeds for a mathematical definition of squares and rectangles. ZDM, 47(3), 391-405. https://doi.org/10.1007/s11858014-0636-5.

Battista, M. T. (2007). The development of geometric and spatial thinking. In F. K. Lester (Hrsg.), Second handbook of research on mathematics teaching and learning. A project of the National Council of Teachers of Mathematics (S. 843-909). Charlotte: Information Age Publishing.

Burger, W.F., \& Shaughnessy, J. (1986). Characterizing the van Hiele levels of development in geometry. Journal for Research in Mathematics Education, 17(1), 31-48. https://doi.org/10.2307/749317.

Chen, Z., \& Siegler, R. S. (2000). Across the great divide: Bridging the gap between understanding of toddlers' and older children's thinking. Monographs of the Society for Research in Child Development, 65(2), v-96. https://doi.org/10.1111/1540-5834.00072.

Clements, D.H., Swaminathan, S., Zeitler, H. M. A., \& Sarama, J. (1999). Young children's concepts of shape. Journal for Research in Mathematics Education, 30(2), 192-212.

Cohen, J. (1988). Statistical Power Analysis for the Behavioral Sciences (2. Aufl.). Hillsdale: Lawrence Erlbaum.

Collins, D. (2003). Pretesting survey instruments: an overview of cognitive methods. Quality of Life Research, 12(3), 229-238. https://doi.org/10.1023/A:1023254226592.

Eid, M., Gollwitzer, M., \& Schmitt, M. (2015). Statistik und Forschungsmethoden: mit Online-Materialien. Weinheim: Beltz.

Franke, M., \& Reinhold, S. (2016). Didaktik der Geometrie. In der Grundschule (3. Aufl.). Berlin, Heidelberg: Springer Spektrum.

Fujita, T. (2012). Learners' level of understanding of the inclusion relations of quadrilaterals and prototype phenomenon. Journal of Mathematical Behavior, 31(1), 60-72. https://doi.org/10.1016/j.jmathb. 2011.08.003.

Fujita, T., \& Jones, K. (2007). Learners' understanding of the definitions and hierarchical classification of quadrilaterals: towards a theoretical framing. Research in Mathematics Education, 9(1), 3-20. https:// doi.org/10.1080/14794800008520167.

Fuys, D., Geddes, D., \& Tischler, R. (1988). The van Hiele model of thinking in geometry among adolescents. Reston: National Council of Teachers of Mathematics.

Gagatsis, A., Sriraman, B., Elisa, I., \& Modestou, M. (2006). Exploring young children's geometrical strategies. Nordic Studies in Mathematics Education, 11(2), 23-50.

Grassmann, M. (1996). Geometrische Fähigkeiten der Schulanfänger. Grundschulunterricht, 43(5), 25-27. 
Grassmann, M., Klunter, M., Köhler, E., Mirwald, E., Raudies, M., \& Thiel, O. (2002). Mathematische Kompetenzen von Schulanfängern. Teil 1: Kinderleistungen - Lehrererwartungen. Potsdam: Universitätsverlag.

Gunčaga, J., \& Žilková, K. (2019). Visualisation as a method for the development of the term rectangle for pupils in primary school. European Journal of Contemporary Education, 8(1), 52-68. https://doi. org/10.13187/ejced.2019.1.52.

Gunčaga, J., Tkačik, Š., \& Žilková, K. (2017). Understanding of selected geometric concepts by pupils of pre-primary and primary level education. European Journal of Contemporary Education, 6(3), 497-515.

Halat, E., \& Yeşil-Dağlı, Ü. (2016). Preschool students' understanding of a geometric shape, the square. Bolema, 30(55), 830-848. https://doi.org/10.1590/1980-4415v30n55a25.

Hannibal, M. A. (1999). Young children's developing understanding of geometric shapes. Teaching Children Mathematics, 5(6), 353-357.

Heinze, A. (2002a). „,.. aber ein Quadrat ist kein Rechteck“ - Schülerschwierigkeiten beim Verwenden einfacher geometrischer Begriffe in Jahrgang 8. ZDM, 34(2), 51-55.

Heinze, A. (2002b). "... because a square is not a rectangle"-Students' knowledge of simple geometrical concepts when starting to learn proof. In A. Cockburn \& E. Nardi (Hrsg.), Proceedings of the 26th Conference for the Psychology of Mathematics Education (Bd. 3, S. 81-88). United Kingdom: Norwich.

Hischer, H. (2012). Grundlegende Begriffe der Mathematik. Entstehung und Entwicklung. Wiesbaden: Vieweg+Teubner.

van Hiele, P. M. (1986). Structure and insight. A theory of mathematics education. Orlando: Academic Press.

Höglinger, S., \& Senftleben, H.-G. (1997). Schulanfänger lösen geometrische Aufgaben. Grundschulunterricht, 44(5), 36-39.

Hull, C.L. (1920). Quantitative aspects of evolution of concepts. An experimental study. Psychological Monographs, 28(1), i-86. https://doi.org/10.1037/h0093130.

Koleza, E., \& Giannisi, P. (2013). Kindergarten children's reasoning about basic geometric shapes. In B. Ubuz, C. Haser \& M. A. Mariotti (Hrsg.), Proceedings of the eighth congress of the European society for research in mathematics education. CERME 8. (S. 2118-2127). Ankara: Middle East Technical University and ERME.

Kürpig, F., \& Niewiadomski, O. (1992). Grundlehre Geometrie. Begriffe, Lehrsätze, Grundkonstruktionen. Wiesbaden: Vieweg+Teubner.

Kyriakides, L. (1999). Research on baseline assessment in mathematics at school entry. Assessment in Education, 6(3), 357-375.

Lehrer, R., Jenkins, M., \& Osana, H. (1998). Longitudinal study of children's reasoning about space and geometry. In R. Lehrer \& D. Chazan (Hrsg.), Designing learning environments for developing understanding of geometry and space (S. 137-167). New Jersey: Lawrence Erlbaum.

Levenson, E., Tirosh, D., \& Tsamir, P. (2011). Preschool geometry. Theory, research and practical perspectives. Rotterdam: Sense Publishers.

Ma, H.L., Lee, D. C., Lin, S.H., \& Wu, D. B. (2015). A study of Van Hiele of geometric thinking among 1 st through 6 th Graders. Eurasia Journal of Mathematics, Science and Technology Education, 11(5), 1181-1196. https://doi.org/10.12973/eurasia.2015.1412a.

Maier, A.S. (2019). Geometrisches Begriffsverständnis von 4- bis 6-jährigen Kindern in Deutschland und England. Münster: Waxmann.

Medin, D. L., \& Smith, E. E. (1984). Concepts and concept formation. Annual review of psychology, 35(1), 113-138.

Niedersächsisches Kultusministerium (2017). Kerncurriculum für die Grundschule Schuljahrgänge 1-4. Mathematik. http://db2.nibis.de/1db/cuvo/datei/0003_gs_mathe_56.pdf. Zugegriffen: 5. Juli 2017.

Piaget, J., Inhelder, B., \& Szeminska, A. (1974). Die natürliche Geometrie des Kindes. Stuttgart: Klett.

Razel, M., \& Eylon, B.-S. (1991). Developing mathematics readiness in young children with the Agam Programm. Paper presented at the meeting of the Fifteenth Conference of the International Group for the Psychology of Mathematics Education, Genua.

Reemer, A., \& Eichler, K.-P. (2005). Vorkenntnisse von Schulanfängern zu geometrischen Begriffen. Grundschulunterricht, 11, 37-42.

Rosch, E.H. (1978). Principles of categorization. In E.H. Rosch \& B. B. Lloyd (Hrsg.), Cognition and categorization (S. 27-48). Hillsdale, New Jersey: Lawrence Erlbaum.

Satlow, E., \& Newcombe, N. (1998). When is a triangle not a triangle? Young children's developing concepts of geometric shape. Cognitive Development, 13(4), 547-559. 
Schmid, H.-J. (2000). Methodik der Prototypentheorie. In M. Mangasser-Wahl (Hrsg.), Prototypentheorie in der Lingusistik. Anwendungsbeispiele - Methodenreflexion - Perspektiven (S. 49-72). Tübingen: Stauffenberg.

Siegler, R. S. (2004). U-shaped interest in U-shaped development-and what it means. Journal of Cognition and Development, 5(1), 1-10. https://doi.org/10.1207/s15327647jcd0501_1.

Siegler, R. S. (2006). Microgenetic analyses of learning. In D. Kuhn, R. S. Siegler, W. Damon \& E. M. Lerner (Hrsg.), Cognition, perception, and language 6. Aufl. Handbook of child psychology, (Bd. 2, S. 464-510). New Jersey: Wiley.

Strauss, S. (1982). Introduction. In S. Strauss (Hrsg.), U-shaped behavioral growth (S. 1-9). : Academic Press.

Tsamir, P., Tirosh, D., \& Levenson E. (2008). Intuitive nonexamples: the case of triangles. Educational Studies in Mathematics, 69(2), 81-95.

Unterhauser, E. (2020). Geometrisches Begriffsverständnis in der frühen Bildung. Eine Interviewstudie zu den Begriffen Vier- und Dreieck bei Kindergartenkindern. Wiesbaden: Springer.

Unterhauser, E., \& Gasteiger, H. (2018). Verständnis des geometrischen Begriffs Viereck bei Kindern zwischen vier und sechs Jahren. Frühe Bildung, 7(3), 152-158. https://doi.org/10.1026/2191-9186/ a000382.

Vinner, S., \& Hershkowitz, R. (1980). Concept images and common cognitive paths on the development of some simple geometrical concepts. In R. Karplus (Hrsg.), Proceedings of the Fourth International Conference for the Psychology of Mathematics Education (S. 177-184). Berkley: Lawrence Hall of Science.

Vollrath, H.-J. (1984). Methodik des Begriffslehrens im Mathematikunterricht. Stuttgart: Klett.

Wellstein, H., \& Kirsche, P. (2009). Elementargeometrie. Eine aufgabenorientierte Einführung. Wiesbaden: Vieweg+Teubner.

Winter, H. (1983). Über die Entfaltung begrifflichen Denkens im Mathematikunterricht. Journal für Mathematik-Didaktik, 4(3), 175-204. https://doi.org/10.1007/BF03339230.

Wöller, S. (2020). Individuelle Begriffskonzepte von 8- bis 12-Jährigen über Würfel und Quader. Hamburg: Dr. Kovač.

Yin, H. S. (2003). Young children's concept of shape: Van Hiele visualization level of geometric thinking. The Mathematics Educator, 7(2), 71-85.

Žilková, K., \& Kopáčová, P. (2018). Young children's concepts of geometric shapes. Harlow: Pearson. 